\title{
Untuned antiviral immunity in COVID-19 revealed by temporal type I/III interferon patterns and flu comparison
}

\author{
loanna-Evdokia Galani ${ }^{1,8}$, Nikoletta Rovina2,8, Vicky Lampropoulou1, Vasiliki Triantafyllia®1, \\ Maria Manioudaki', Eleftherios Pavlos ${ }^{1}$, Evangelia Koukaki ${ }^{3}$, Paraskevi C. Fragkou $\mathbb{1}^{4}$, Vasiliki Panou ${ }^{3}$, \\ Vasiliki Rapti ${ }^{4}$, Ourania Koltsida ${ }^{5}$, Andreas Mentis ${ }^{6}$, Nikolaos Koulouris ${ }^{3}$, Sotirios Tsiodras ${ }^{4}$, \\ Antonia Koutsoukou ${ }^{2}$ and Evangelos Andreakos ${ }^{1,7 凶}$
}

A central paradigm of immunity is that interferon (IFN)-mediated antiviral responses precede pro-inflammatory ones, optimizing host protection and minimizing collateral damage $^{1,2}$. Here, we report that for coronavirus disease 2019 (COVID-19) this paradigm does not apply. By investigating temporal IFN and inflammatory cytokine patterns in 32 moderate-to-severe patients with COVID-19 hospitalized for pneumonia and longitudinally followed for the development of respiratory failure and death, we reveal that IFN- $\lambda$ and type I IFN production were both diminished and delayed, induced only in a fraction of patients as they became critically ill. On the contrary, pro-inflammatory cytokines such as tumor necrosis factor (TNF), interleukin (IL)-6 and IL-8 were produced before IFNs in all patients and persisted for a prolonged time. This condition was reflected in blood transcriptomes wherein prominent IFN signatures were only seen in critically ill patients who also exhibited augmented inflammation. By comparison, in 16 patients with influenza (flu) hospitalized for pneumonia with similar clinicopathological characteristics to those of COVID-19 and 24 nonhospitalized patients with flu with milder symptoms, IFN- $\lambda$ and type I IFN were robustly induced earlier, at higher levels and independently of disease severity, whereas pro-inflammatory cytokines were only acutely produced. Notably, higher IFN- $\lambda$ concentrations in patients with COVID-19 correlated with lower viral load in bronchial aspirates and faster viral clearance and a higher IFN- $\lambda$ to type I IFN ratio correlated with improved outcome for critically ill patients. Moreover, altered cytokine patterns in patients with COVID-19 correlated with longer hospitalization and higher incidence of critical disease and mortality compared to flu. These data point to an untuned antiviral response in COVID-19, contributing to persistent viral presence, hyperinflammation and respiratory failure.

COVID-19, triggered by the betacoronavirus SARS-CoV-2, has become one of the worst pandemics of our time, causing high incidence of pneumonia, acute respiratory distress syndrome (ARDS) and death ${ }^{3,4}$. One of the most notable features of SARS-CoV-2 infection is that it goes unnoticed for a remarkably prolonged period of time, running a course of a mild or uncomplicated illness for weeks until sudden and severe symptoms develop in a subgroup of patients, requiring hospitalization, oxygen support and/or admission to an intensive care unit (ICU) $)^{3,4}$. This scenario is consistent with an unusually long incubation period of the virus, ranging from 2 to $14 \mathrm{~d}$ and an unusually long presence of virus in the respiratory tract, often being detectable for over a month after initial infection by conventional molecular diagnostic tests ${ }^{5,6}$. By comparison, influenza virus infection, the main respiratory virus accounting for pneumonia hospitalizations until now, has an incubation time of 1 to $4 \mathrm{~d}$, a short window of virus positivity of a few days and an abrupt onset of symptoms causing pneumonia within $1-3 \mathrm{~d}^{7,8}$. Other frequent respiratory viruses such as respiratory syncytial viruses, rhinoviruses, parainfluenza viruses, metapneumonoviruses and common cold coronaviruses have also shorter incubation times (ranging from 1-5d) and more rapid and acute manifestation of symptoms ${ }^{9}$, rendering SARS-CoV-2 quite unique in that respect. The basis of this difference is unknown but is likely to be a key driver of the pathophysiology of COVID-19 underlying its distinctive disease course and clinical manifestations.

The hallmark of COVID-19 is the development of a hyper-inflammatory response, also known as 'cytokine storm', impairing the gas-exchange function and leading to ARDS, multi-organ failure and death ${ }^{10-12}$. We and others have previously shown that a finely tuned antiviral response, orchestrated by IFN- $\lambda$ (type III IFN) and type I IFN is critical for balancing immunity for optimal protection and minimal damage ${ }^{13-15}$. Deviation from this balance can unleash a detrimental 'cytokine storm' with devastating consequences for human health. A recent study suggested that in patients with COVID-19, type I IFN and IFN- $\lambda$ are not produced as they could not be detected in the sera of a small COVID-19 cohort of otherwise unspecified clinical characteristics ${ }^{16}$. In contrast, another study reported that type I IFN is induced in patients with COVID-19 and indicated that its concentration might be reduced

\footnotetext{
'Laboratory of Immunobiology, Center for Clinical, Experimental Surgery and Translational Research, Biomedical Research Foundation of the Academy of Athens, Athens, Greece. ${ }^{2} \mathrm{CCU}$, 1st Department of Respiratory Medicine, National and Kapodistrian University of Athens, Medical School, 'Sotiria' General Hospital of Chest Diseases, Athens, Greece. ${ }^{3} 1$ st Department of Respiratory Medicine, National and Kapodistrian University of Athens, Medical School, 'Sotiria' General Hospital of Chest Diseases, Athens, Greece. ${ }^{4} 4$ th Department of Internal Medicine, Attikon University Hospital, University of Athens Medical School, Athens, Greece. ${ }^{52}$ nd Respiratory Clinic, 'Sotiria' General Hospital of Chest Diseases, Athens, Greece. ${ }^{6}$ Department of Microbiology, Hellenic Pasteur Institute, Athens, Greece. ${ }^{7}$ Airway Disease Infection Section, National Heart and Lung Institute, Imperial College London, London, UK. ${ }^{8}$ These authors contributed equally to this work: Ioanna-Evdokia Galani, Nikoletta Rovina. ${ }_{e}$-mail: vandreakos@bioacademy.gr
} 
in those that are critically ill ${ }^{17}$. Such discrepancy could be due to the fact that each of these studies focuses on a single and likely distinct snapshot of an apparently heterogeneous disease process. Therefore, pursuing kinetic analyses is pertinent to delineating the course of the immune response, especially given that cytokines are transiently produced. This criterion is particularly true for IFNs, which are expressed early during infection and are rapidly downregulated thereafter.

Here, we have performed a comprehensive temporal analysis of type I and type III IFNs and major inflammatory cytokine patterns in 32 patients with COVID-19 and 16 patients with flu hospitalized for community-acquired pneumonia and longitudinally followed up according to current World Health Organization guidelines ${ }^{18}$. Both groups of patients exhibited similar clinicopathological characteristics and comparable disease severity on admission (Supplementary Table 1). We have also analyzed 24 patients with milder cases of flu with no radiological findings of pneumonia and no need for hospitalization (referred to as mild flu; Supplementary Table 1), as well as 10 healthy individuals. Using high-sensitivity Luminex and ELISA assays, we quantified 18 cytokines and chemokines relevant to antiviral immunity and hyperinflammation in patient serum collected at defined time intervals following hospital admission (Fig. 1a and Extended Data Fig. 1a). This analysis aligns patients on the basis of the same clinical criteria of disease symptoms and severity, mainly the presence of pneumonia and the requirement for oxygen support.

We found that patients with COVID-19 had profoundly impaired induction of both IFN- $\lambda$ and type I IFNs. IFN- $\lambda$ and type I IFNs were not detectable in most patients with COVID-19 (with median levels at the limit of quantification of the assay), although some patients made IFN- $\lambda$ and fewer of them also made IFN- $\alpha$ (Fig. 1b). This observation contrasts patients with flu who almost uniformly expressed both types of IFNs, within the first (day 1-3) time interval of admission and at significantly higher concentrations. At all cases, IFN expression was transient, with type I IFN levels rapidly declining after the first $3 \mathrm{~d}$ of hospitalization, whereas IFN- $\lambda$ persisted for longer. Notably, despite their limited ability to make IFNs, patients with COVID-19 robustly expressed pro-inflammatory cytokines such as TNF, IL-6, IL-7, IL-8, IL-10, IFN- $\gamma$ and CCL3 that were maintained at high concentrations for a prolonged time (Fig. 1b). Other cytokines such as IL-1 $\beta$, IL-12, IL-23 and CCL4 were also significantly upregulated at specific time intervals compared to healthy individuals, reflecting the heterogeneity of the disease course (Extended Data Fig. 2).

A similar pattern emerged when comparisons were made according to disease symptom onset (Extended Data Fig. 1b). Patients with COVID-19 exhibited markedly delayed and reduced IFN- $\lambda$ and type I IFN levels, which were detectable only in a fraction of the patients and from days 7-10 onwards of symptom onset (Extended Data Fig. 3a,b). By comparison, all patients with flu exhibited high levels of these cytokines during the first $6 \mathrm{~d}$ (Extended Data Fig. 3a,b). Although patients with COVID-19 made little IFN during the first $6 \mathrm{~d}$ of symptom onset, they potently produced pro-inflammatory cytokines and chemokines such as TNF, IL-6, IL-8, IL-10 and CCL3 at concentrations similar to flu (Extended Data Fig. 3b,c). Moreover, they exhibited prolonged expression of pro-inflammatory mediators, with high concentrations of TNF, IL-6, IL-7, IL-8, IL-10 and CCL4 remaining detectable for over 3 weeks of onset, whereas in patients with flu a number of these were by that time downregulated.

Notably, patients with COVID-19 were admitted to hospital with similar markers of systemic inflammation such as C-reactive protein (CRP) concentrations, white blood cell (WBC) and neutrophil counts and neutrophil/lymphocyte (N/L) ratio to patients with flu (Supplementary Table 1 and Extended Data Fig. $4 a-f)$. They even had lower fever and a lower CURB-65 score, a commonly used measure of pneumonia severity ${ }^{19}$ (Extended Data Fig. 4g,h). However, during follow up, patients with COVID-19 developed a much higher incidence of ARDS requiring ICU support. In our cohort, 16 out of 32 patients (50\%) developed critical disease, 3 of whom died, compared to only 3 out of 16 patients with flu (18.7\%), none of whom died (Extended Data Fig. 5). Patients with COVID-19 became critically ill over a much broader time window (with the first patient onset at day 1 and the last at day 9 after hospital admission; Fig. 1a and Extended Data Fig. 5) than patients with flu, who manifested critical disease within the first day after admission. This finding is in agreement with the high incidence and protracted course of severe respiratory failure described for COVID-19 (refs. ${ }^{4,12}$ ). Interestingly, among patients with COVID-19, those who became critically ill had higher CRP concentrations, WBC and neutrophil counts and N/L ratio on admission (Extended Data Fig. 4a-f), but not CURB65 or fever (Extended Data Fig. 4g,h and Supplementary Table 2). Critically ill patients with flu also had a tendency for higher WBC and neutrophil counts, N/L ratio and CURB-65, whereas nonhospitalized patients with flu did not exhibit any of these increases (Extended Data Fig. 4a-h).

We thus examined whether temporal cytokine patterns differ between the various patient groups. Notably, we observed that although patients with COVID-19 that did not become critically ill produced little type I or III IFN, the ones that became critically ill had IFN- $\lambda$ levels that were significantly higher at the day 1-3 time interval compared to healthy and noncritically ill patients (Fig. 2a). Some of the critically ill patients also made IFN- $\alpha$ (Fig. 2a), albeit at significantly lower amounts compared to nonhospitalized patients with mild flu (Fig. 2a) or the total number of hospitalized patients with flu (both critically and noncritically ill; $P<0.05$ ). On the contrary, all patients with COVID-19 made pro-inflammatory cytokines such as TNF, IL-6, IL-8, IL-10 and IFN- $\gamma$, with critically ill patients exhibiting also significantly higher concentrations of IL- 6 and IL-7 than noncritically ill patients at specific time intervals and a tendency for higher IFN- $\gamma$, consistent with the increased hyper-inflammatory state they were in (Fig. 2a and Extended Data Fig. 6). Individual patient data further confirmed these trends (Extended Data Fig. 7). CCL3 was significantly higher than healthy controls in noncritically ill patients with COVID-19 but not in those that were critically ill (Fig. 2a). By comparison, critically ill and noncritically ill patients with flu did not differ in their ability to make type I and type III IFNs nor pro-inflammatory cytokines such as TNF, IL-6 or IL-7 (Fig. 2a and Extended Data Fig. 6). Similarly, nonhospitalized patients with flu with mild disease exhibited strong production of type I and type III IFNs, indicating that across the spectrum of flu disease severity the antiviral response remains robust. They also exhibited similar production of pro-inflammatory cytokines such as TNF, IL-6, IL-7, IL-8 and IFN- $\gamma$ but higher levels of CCL3 compared to either noncritically or critically ill hospitalized patients with flu. Visualizing these patterns on radar plot reveals a major imbalance in the induction of antiviral and pro-inflammatory responses of patients with COVID-19 that does not occur in flu (Fig. 2b).

We next sought to determine whether imbalanced cytokine patterns in patients with COVID-19 are related to systemic immune effects and parameters linked to disease severity. To that end, we obtained temporal WBC transcriptomes from five healthy individuals and nine patients with COVID-19, five noncritically and four critically ill, starting from day 1 of entry to the ward or ICU and at different time points thereafter. In total, 24 comprehensive RNA-seq gene expression datasets were analyzed. Clustering analyses revealed that samples group according to the severity of the clinical phenotype, indicating this as the main source of variation and providing a way to predict which patients will progress to develop critical or noncritical illness (Fig. 3a and Extended Data Fig. 8). Focusing at day 1 as the most relevant time point, we found that 4,225 genes were differentially expressed in patients with COVID-19 compared to healthy individuals (Supplementary Table 3). When 

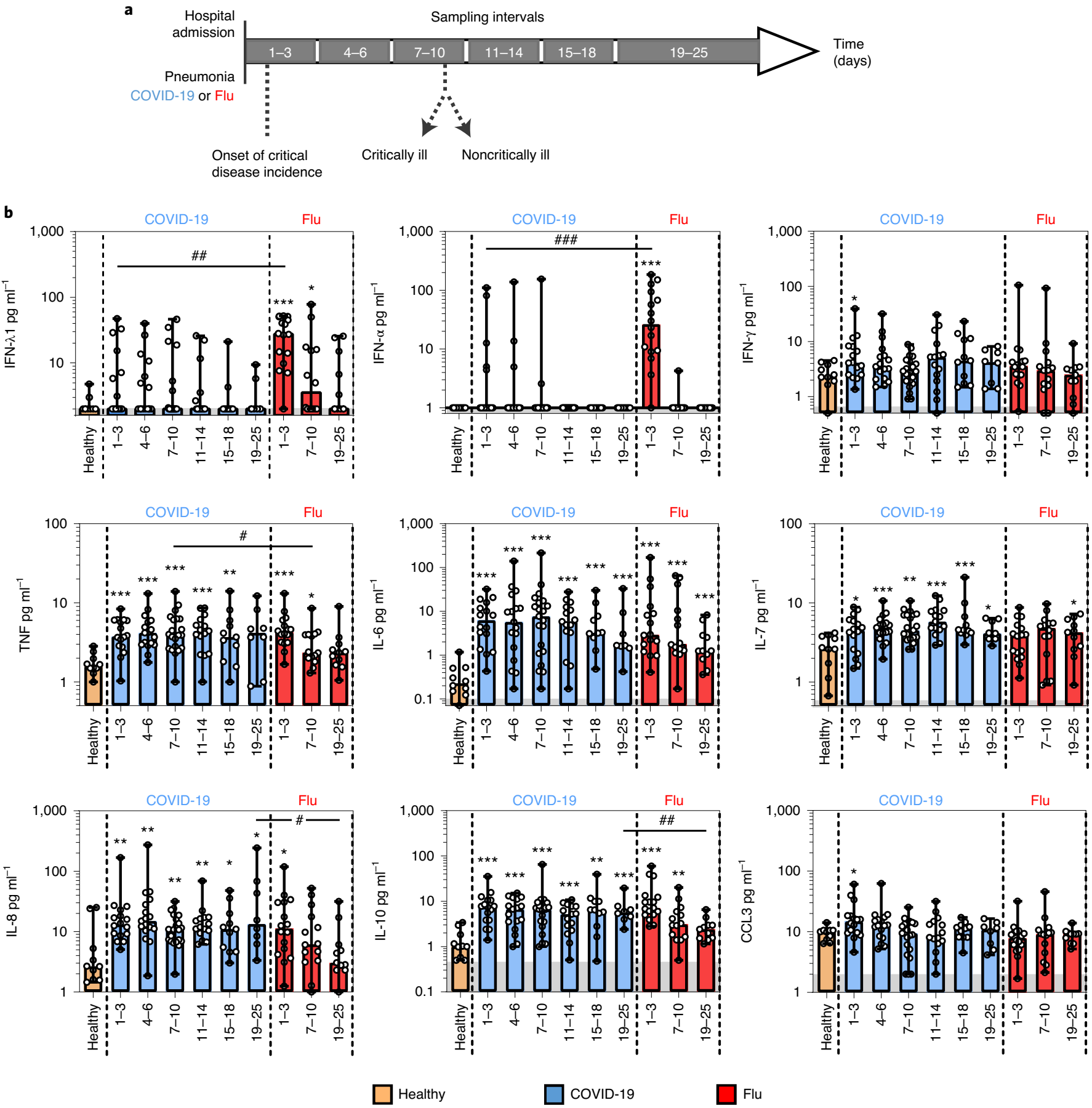

Fig. 1 | Temporal IFN and inflammatory cytokine patterns of patients with COVID-19 and flu in relation to hospital admission. a, Schematic showing the experimental design with sampling at specific time intervals after hospital admission of 32 patients with COVID-19 and 16 patients with flu with pneumonia followed longitudinally. Dashed lines indicate the time of the first and last onset of critical disease, respectively. $\mathbf{b}$, Serum levels of IFN- $\lambda 1$, IFN- $\alpha$, IFN- $\gamma$, TNF, IL-6, IL-7, IL-8, IL-10 and CCL3 at various time intervals after hospital admission. Data are presented as scatter plots with dots showing individual patient measurements, columns median values and error bars the range. For COVID-19, $n=16,17,21,15,11$ and 8 for each of the six consecutive time intervals. For flu, $n=16,14$ and 11, respectively. For healthy, $n=10$. Gray shading marks the limit of quantification of the assay. $P$ values were determined by a two-tailed Mann-Whitney $U$-test for nonparametric comparisons. ${ }^{\star} P<0.05,{ }^{\star \star} P<0.01$ and ${ }^{\star \star \star}{ }^{\star} P<0.001$ show significance over healthy controls. ${ }^{\sharp} P<0.05,{ }^{\# \#} P<0.01$ and ${ }^{\# \#} P<0.001$ show significance between COVID-19 and flu groups.

critically and noncritically ill patients were compared separately to healthy controls, 4,214 and 4,902 differentially expressed genes (DEGs) were observed, respectively, of which 1,979 were common whereas the rest were uniquely found in one or the other patient group (Fig. 3b and Supplementary Tables 3 and 4). Of these DEGs, 2,674 genes were also significantly different between critically and noncritically ill patients (Supplementary Table 5). Volcano plots pointed out notable differences in the most highly regulated genes between the groups, with critically ill patients exhibiting stronger immune and antiviral response gene patterns (Fig. 3c-e). Pathway analysis of DEGs indeed revealed that the most significant pathways over-represented in critically ill patients were related to the 
positive regulation of the immune system, the activation of the innate immune response, the defense response to virus and the cellular response to IFN (Fig. 3f and Supplementary Table 6). Induction of the IL-1 $\beta$ production pathway and response to IL- 1 were also prominent. By contrast, in noncritically ill patients these pathways were not significantly upregulated with the exception of the IL-1 $\beta$ production pathway (Fig. 3f). Other pathways over-represented instead included the regulation of the cellular component size and natural killer (NK) cell cytotoxicity (Fig. 3f).

Accordingly, heat maps with temporal information unveiled strong induction of a long set of antiviral genes in critically ill patients compared to only a fraction of them being upregulated in the noncritically ill group (Fig. 4a and Supplementary Table 3). This set includes classical antiviral response genes, which can be induced by type I and III IFN, such as Stat1, Stat3, Irf1, Irf2, Socs3, Isg20, Oasl, Ifi5, Ifit1b, Ifit5, Ifitm10, Gbp1, Gbp2, Gbp4, Gbp5 and Gbp6, all significantly upregulated in critically ill patients over noncritically ill or healthy individuals and Irf3, Socs3, Mx1, Oas1, Ifi27, Ifi44 and Ifitm 5 also upregulated in noncritically ill patients albeit to a lower extent than in critically ill patients (Supplementary Table 5). Comparison of the total set of antiviral genes expressed at day 1 as shown in Fig. 4a confirmed a statistically significant difference between critically and noncritically ill patients $\left(P=1.25 \times 10^{-20}\right)$, in agreement with the distinct patterns of production of IFN- $\lambda$ and type I IFNs in these patients. This stronger type I/III IFN response in critically ill patients was unlikely to be due to higher expression of IFN receptor components, as no significant differences were observed between Ifnlr1, Il10rb and Ifnar1 mRNA levels among patient groups and healthy individuals except a twofold increase of Ifnar2 in critically ill patients (Supplementary Table 3 ).

Further heat map analyses pointed to a stronger systemic innate immune response in critically ill patients marked by increased expression of key pro-inflammatory mediators, including complement components $(C 2, C 4 b p a)$, cytokines $(C s f 1, C x c l 10)$ and matrix metalloproteinases ( $M m p 8, M m p 9$ ) over healthy individuals (Fig. $4 \mathrm{~b}$ ). On the contrary, noncritically ill patients exhibited enhanced expression of Il10, a major anti-inflammatory protein-dampening cytokine storms and $I l 7$, which is involved in maintaining $\mathrm{T}$ cell homeostasis in the periphery. Notably, inflammasome genes such as Nlrp6, Nrlc4, Nod2, Aim2, Casp9, Casp10, Il1rn and Illr1, all linked to IL- $1 \beta$ production or response ${ }^{20}$, were also upregulated in critically ill patients with COVID-19, whereas Pycard (Asc), a key component of inflammasome assembly, was downregulated in noncritically ill patients over healthy individuals, indicating a prominent role of the IL-1 $\beta$ pathway in more severe disease (Fig. 4c). Pattern-recognition receptors (PRRs) involved in microbial recognition such as $D d x 58$ (Rigi), Aim2, Ifih1 (Mda5), Ifi16, Tlr2 and Tlr4, were also upregulated. Overall, when compared as a set, inflammasome and PRR genes shown in Fig. $4 \mathrm{c}$ were significantly different between the two groups $\left(P=9.72 \times 10^{-7}\right)$. At the cell-type level, gene expression analysis revealed a dominant activated neutrophil/myeloid cell signature (Mpo, Elane, Cd177, Itgam, Arg1, Ceacam8 and Fcgr1a) in the critically ill group that was milder and not significant in noncritically ill patients (Fig. 4d). On the contrary, T, B and NK lymphocyte lineage and related genes (Cd3d, Cd3e, Cd4, Cd8a, Cd19, Cd22 and Ncam1) were markedly downregulated in critically ill patients. These data are consistent with lymphopenia, high neutrophil counts and a high N/L ratio also present in these patients (Extended Data Fig. 4) and previously reported to be associated with more severe disease and worse outcomes in patients with COVID-19 (refs. ${ }^{3,4}$ ). Cytokines such as TNF, IL-6 and IL-8 may directly account for these effects, as they are well known to trigger the mobilization and activation of neutrophils, the development of lymphopenia and the induction of innate immune responses and systemic inflammation ${ }^{21,22}$. Thus, WBC transcriptomes of patients with COVID-19, early during hospitalization, can provide vital information about disease severity and guide treatment such as the administration of IL-1 inhibitors in a more personalized manner.

Interestingly, imbalanced cytokine patterns in patients with COVID-19 with pneumonia were associated with a much worse disease outcome compared to flu. First, the COVID-19 group exhibited higher incidence of critical disease and mortality (Extended Data Fig. 5). Second, patients with COVID-19 overall, as well as when grouped as critically and noncritically ill, required longer hospitalization time than their flu counterparts (Fig. 5a-c). For noncritically and critically ill patients with COVID-19, median time was 14 and $23 \mathrm{~d}$, respectively, compared to flu at 7 and $19 \mathrm{~d}$ (Fig. 5b,c). Prolonged hospitalization could be attributed to the untuned antiviral responses, leading to a more protracted clinical course of COVID-19 relative to flu and a need for longer recovery even for the noncritically ill group.

To identify cytokines and cytokine combinations that can predict hospitalization time, and therefore be of prognostic value for risk stratification independently of known laboratory and clinical severity parameters (such as $\mathrm{O}_{2}$ saturation, respiratory rate or $\mathrm{N} / \mathrm{L}$ ratio), we generated a correlation matrix of the cytokine levels at admission (day 1-3 interval) and the duration of hospital stay (Fig. 4d). We found that higher IL-6 and IL-10, and lower CCL3 concentrations, were directly proportional to the duration of hospitalization (Fig. $5 \mathrm{~d}-\mathrm{f}$ ). The value of IL-6 and IL-10 as biomarkers for monitoring COVID-19 severity has been reported ${ }^{4,23,24}$ but for CCL 3 this is new. Notably, IFN- $\lambda$ concentration also correlated with higher IL- 6 and IL-10 and longer hospitalization time, consistent with their almost exclusive induction in critically but not noncritically ill patients (Fig. 5d).

A question that arises is whether IFN levels induced in critically ill patients are beneficial, as delayed type I or type III IFN production has been shown in animal models to cause immunopathology ${ }^{13,14,25}$ or interfere with epithelial repair ${ }^{26,27}$, respectively. We found that higher IFN- $\lambda$ concentrations during ICU entry were associated with lower SARS-CoV-2 viral load in the respiratory tract and faster viral clearance (Fig. $5 \mathrm{~g}, \mathrm{~h}$ ). Moreover, a higher IFN- $\lambda$ to type I IFN ratio at that time was linked to a shorter stay in the ICU (Fig. 5i), with the two patients with the highest IFN- $\alpha$ levels also exhibiting the longest stay (both $23 \mathrm{~d}$ over a median of $17 \mathrm{~d}$ ). These data suggest that delayed IFN- $\lambda$ induction may still be protective in critically ill patients with COVID-19, whereas IFN- $\alpha$ may do more harm than good, at least in a subset of patients.

Fig. 2 | Comparison of IFN and inflammatory cytokine patterns between subgroups of patients with COVID-19 and flu according to disease severity. a, Serum levels of IFN- $\lambda 1$, IFN- $\alpha$, IFN- $\gamma$, TNF, IL-6, IL-7, IL-8, IL-10 and CCL3 for critically and noncritically ill patients with COVID-19 and flu and nonhospitalized patients with mild flu at days 1-3 and 7-10 time intervals after hospital admission or visit, respectively, as well as healthy individuals. Dots show individual measurements and lines median values of hospitalized patients and healthy individuals. Squares show nonhospitalized patients with flu. Gray shading marks the limit of quantification of the assay. $\mathbf{b}$, Radar plots of median cytokine levels and range of hospitalized patients with COVID-19 and flu developing critical versus noncritical illness, nonhospitalized patients with flu and healthy individuals at the day 1-3 time interval after admission. Each circle in the radar plot represents logarithmically increasing concentrations from 4-256 pg ml-1 as shown in the healthy controls. For days $1-3, n=9,7,24$, 13 and 3 for each of the five consecutive groups, respectively. For days $7-10, n=8,13,15,12$ and 2, respectively. For healthy individuals, $n=10$. $P$ values were determined by a two-tailed Mann-Whitney U-test for nonparametric comparisons. ${ }^{\star} P<0.05,{ }^{\star \star} P<0.01$ and ${ }^{\star \star \star} P<0.001$ show significance over healthy controls. ${ }^{\# P}<0.05,{ }^{\#} P<0.01$ and ${ }^{\# \# P} P<0.001$ show significance between COVID-19 and flu subgroups. 
Taken together, our findings demonstrate that SARS-CoV-2 by the pro-inflammatory process as a second line of protection, it infection does not follow the conventional paradigm of antiviral does the opposite; it triggers the pro-inflammatory response long immunity. Instead of activating first the antiviral response followed before IFN-mediated antiviral defenses are induced, if at all. This
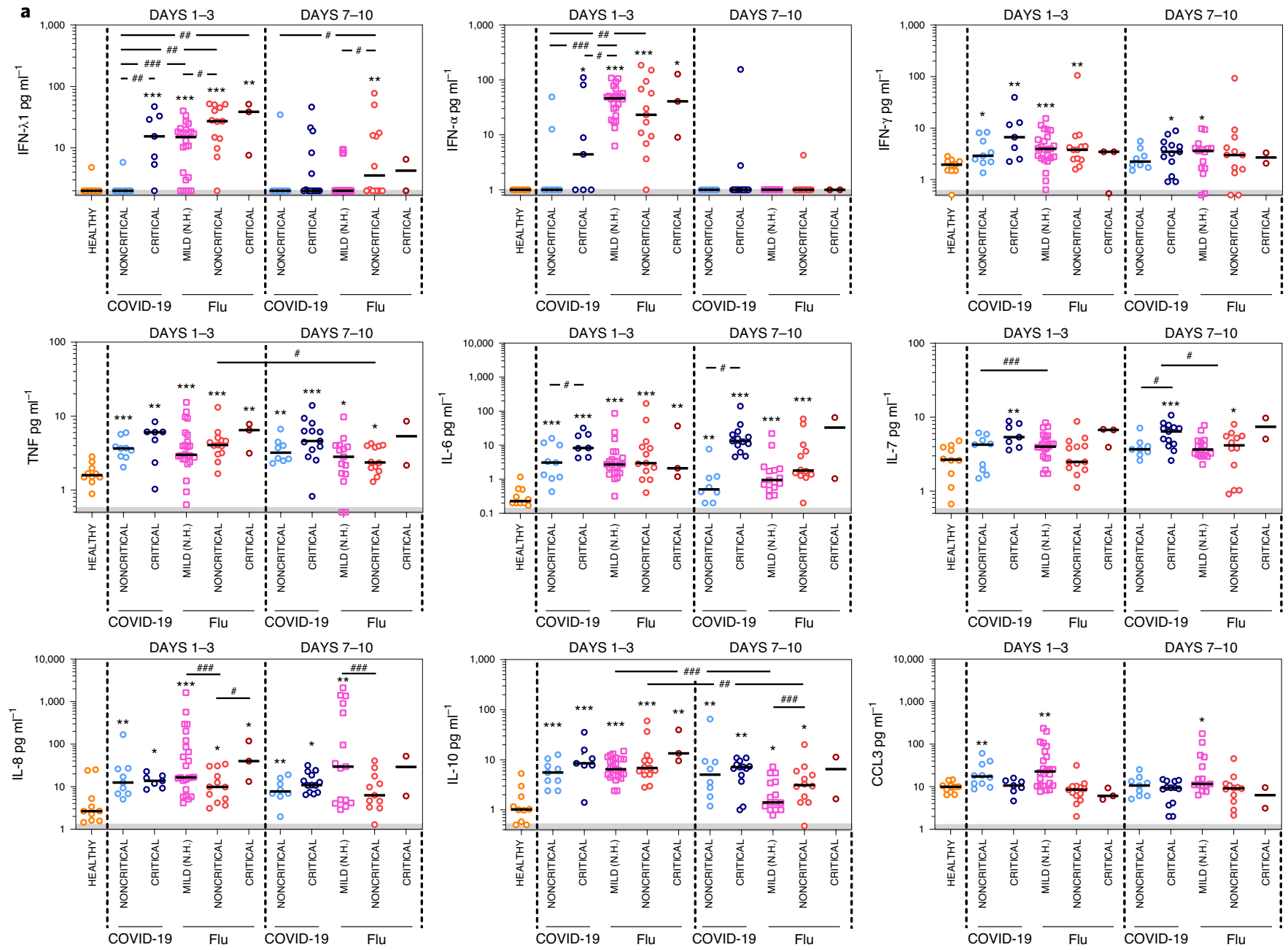

b
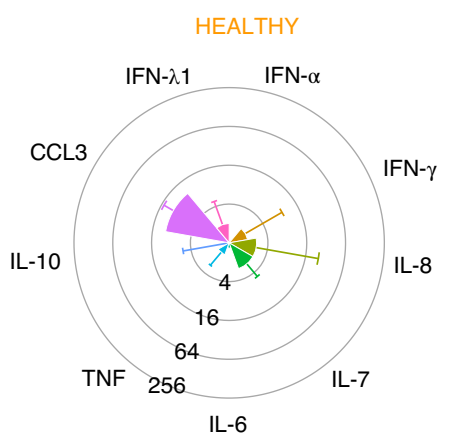

NONCRITICALLY ILL

COVID-19

IFN- $\lambda 1 \quad \alpha$

CRITICALLY ILL
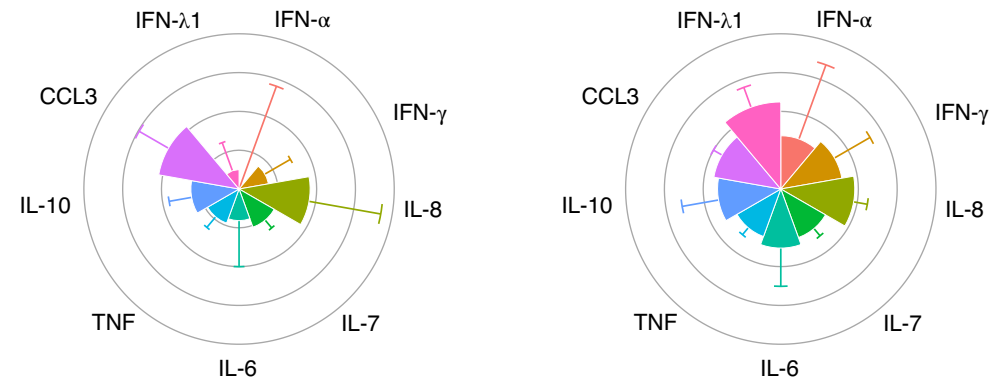

Flu

MILD NONHOSPITALIZED

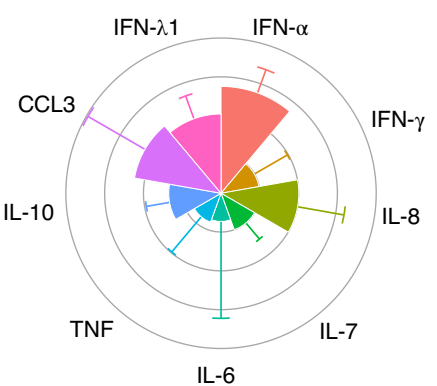

NONCRITICALLY ILL

CRITICALLY ILL
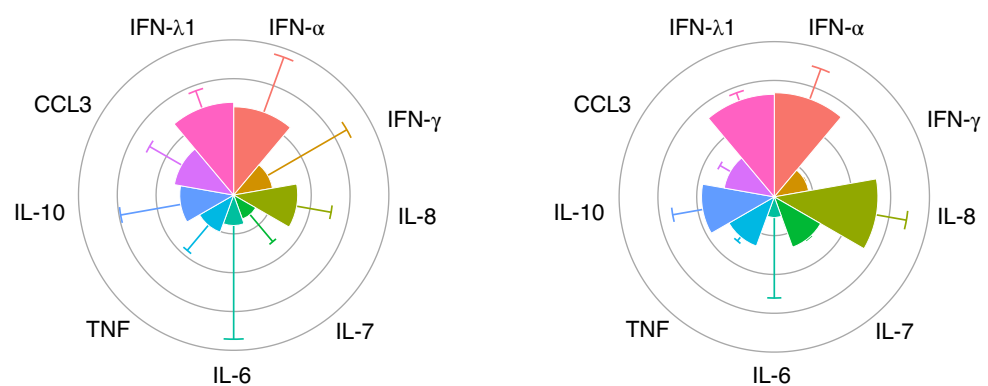


\begin{tabular}{l} 
NONCRITICALLY ILL \\
O Patient 16 \\
O Patient 17 \\
O Patient 18 \\
O Patient 19 \\
O Patient 24 \\
\hline CRITICALLY ILL \\
O Patient 15 \\
O Patient 22 \\
O Patient 23 \\
O Patient 25
\end{tabular}

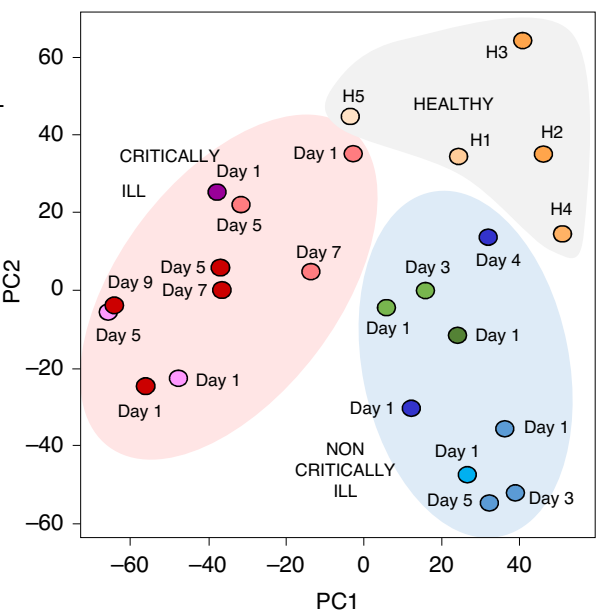

NONCRITICALLY ILL

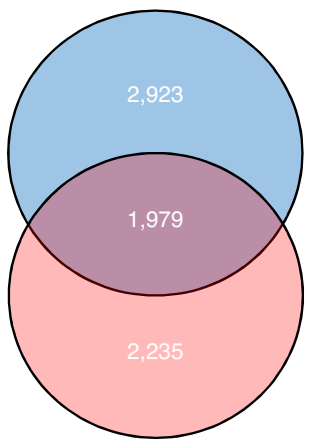

CRITICALLY ILL c

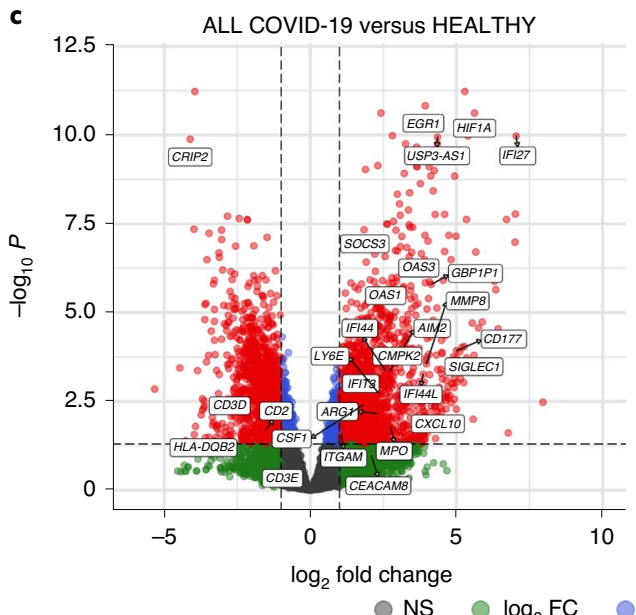

d

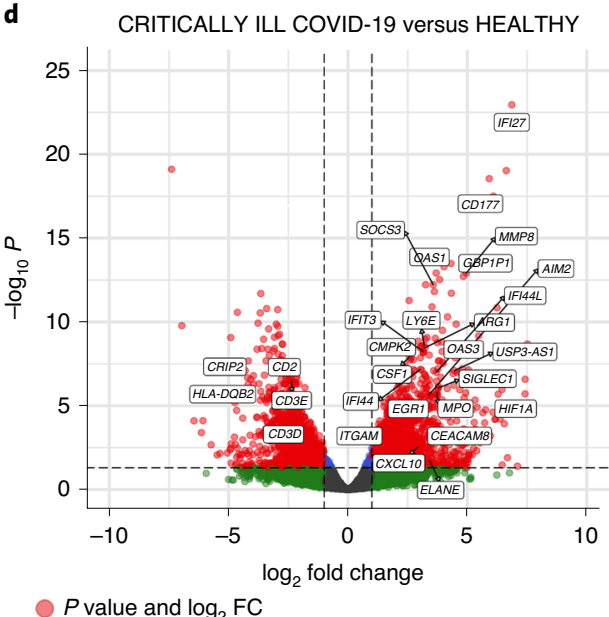

e

NONCRITICALLY ILL COVID-19 versus HEALTHY f
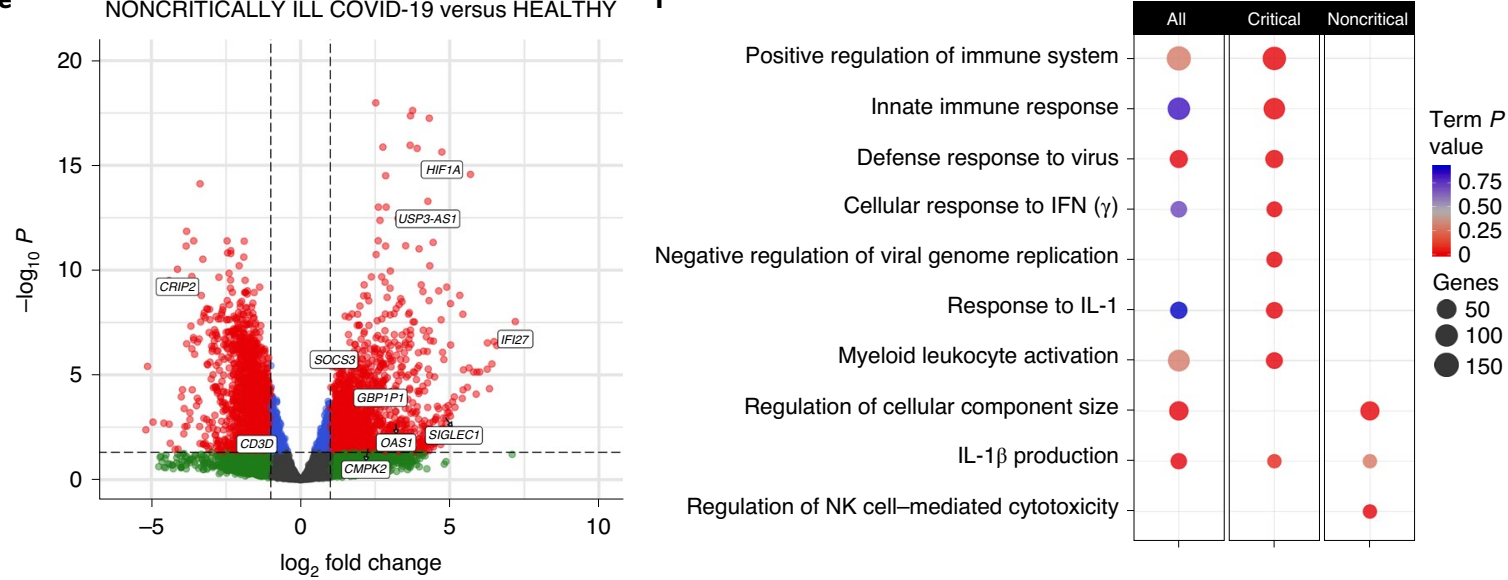

Fig. 3 | Kinetic analysis of blood transcriptional signatures of critically and noncritically ill patients with COVID-19. a, Principal-component analysis of peripheral WBC transcriptomes of critically ill $(n=4)$ and noncritically ill $(n=5)$ patients or healthy $(\mathrm{H} 1-\mathrm{H} 5)$ controls $(n=5)$. b, Venn diagram showing the unique and common DEGs of peripheral WBC transcriptomes from critically ill $(n=4)$ and noncritically ill $(n=5)$ patients compared to healthy $(n=5)$ individuals. c-e, Volcano plots showing the most significantly upregulated and relevant DEGs in all patients with COVID-19 (c) and their critically (d) and noncritically ill (e) subgroups versus healthy individuals at day 1 of hospitalization. DEG analysis was performed using DESeq2 v.1.22.2. FC, fold change; NS, not significant. f, Gene ontology (GO) pathway-enrichment analysis of DEGs of all, critically ill and noncritically ill patients with COVID-19 at day 1 of hospital or ICU entry. ClueGO was employed and term $P$ values were adjusted using Bonferroni correction for multiple comparisons. Visualization was performed using the ggplot2 R package. Dot size indicates the number of genes differentially expressed per pathway, and the red color indicates statistical significance.

scenario is a major paradox and helps explain many of the unique or unusual features of COVID-19. The long virus incubation time and persistence in the respiratory tract, giving positive SARS-CoV-2 tests for weeks, can be attributed to the delayed and/or reduced production of type I and III IFNs. The absent or very mild symptoms of patients for an unusually extended period of time, can be attributed to the lack or impaired and delayed expression of type I IFNs, principal mediators of flu-like disease and symptoms such as runny 


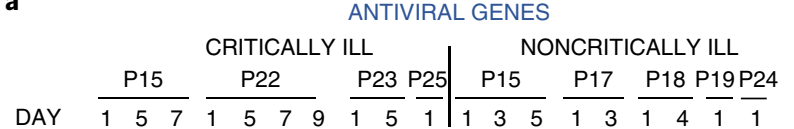
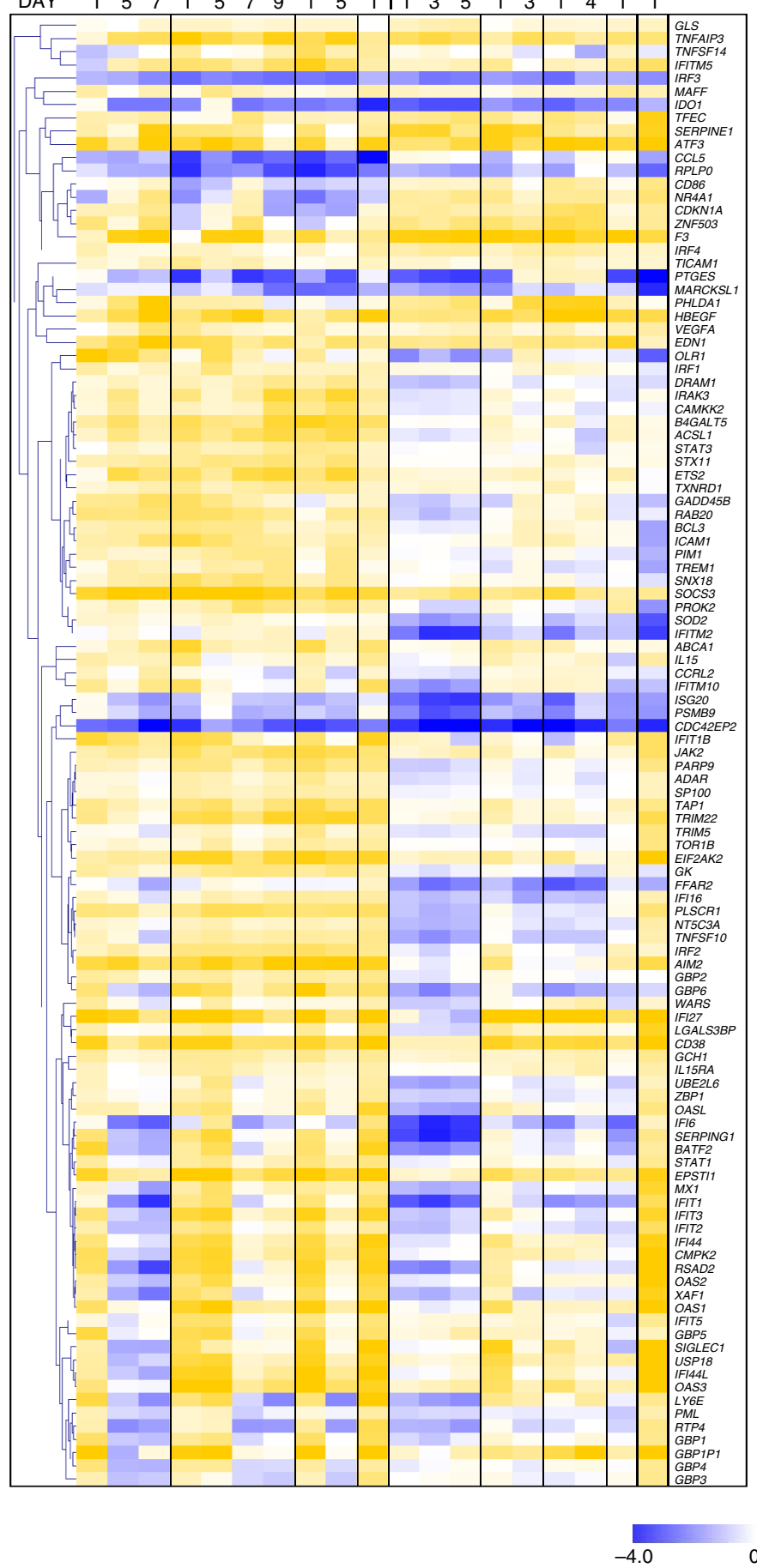

b

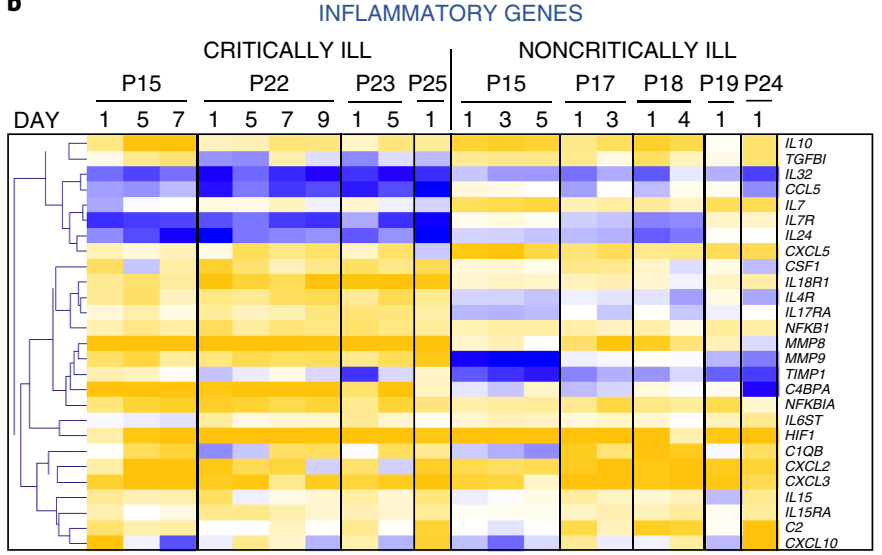

c

INFLAMMASOME \& PATTERN RECOGNITION RECEPTOR GENES

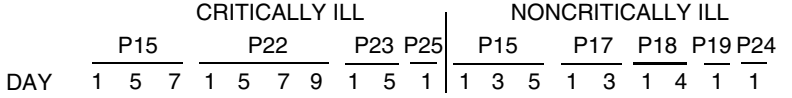

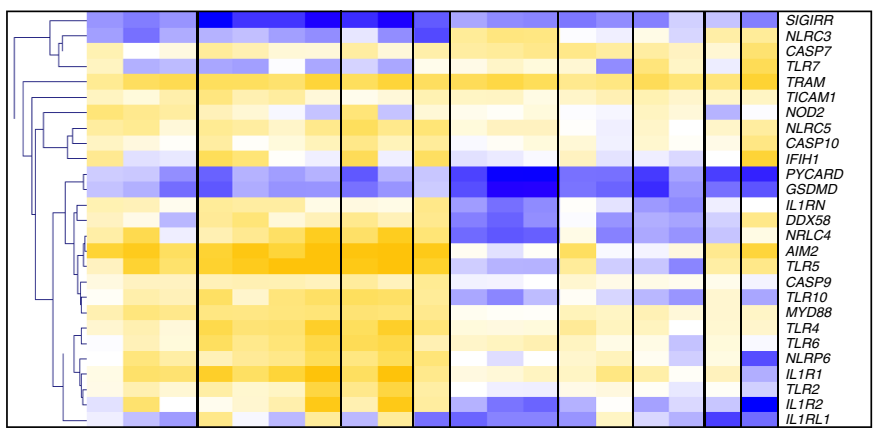

d LEUKOCYTE GENES
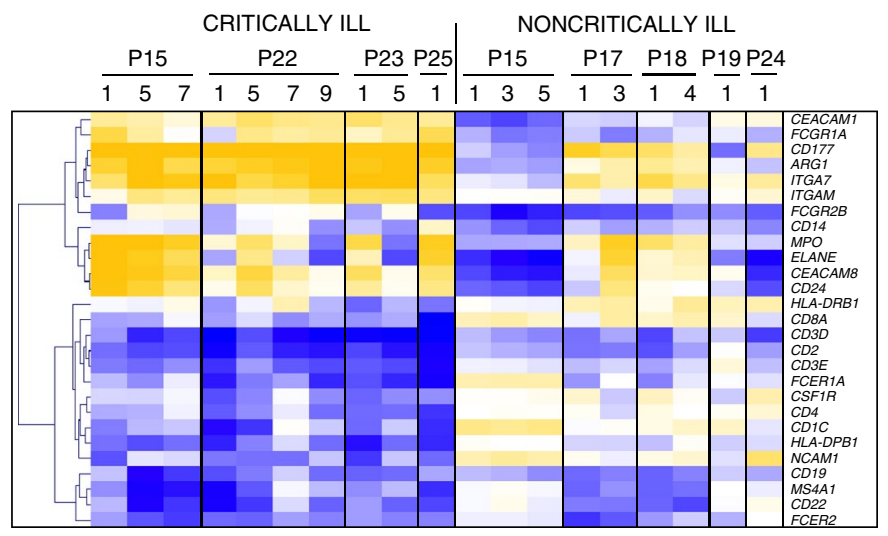

4.0

Fig. 4 | Heat maps of DEGs of critically and noncritically ill patients with COVID-19 compared to healthy individuals. a-d, Heat maps showing temporal patterns of differentially expressed antiviral genes (a), inflammatory genes (b), inflammasome and PRR genes (c) and leukocyte genes (d) of critically ill and noncritically ill patients with COVID-19 compared to healthy individuals. Blue and yellow colors represent down- and upregulated genes, respectively. Data are expressed as $\log _{2}$ fold values over healthy controls. P, patient.

nose, coughing, fatigue, dyspnea and fever in humans ${ }^{28}$. Finally, the early and persistent expression of pro-inflammatory cytokines culminating into prolonged hyperinflammation can promote the sudden development of respiratory failure requiring hospitalization and frequently ICU admission. Noteworthy, in flu the swift induction of the type I and III IFN response, across the spectrum of disease severity, correlates with quicker recovery and markedly lower incidence of critical disease or mortality ${ }^{13,25}$. The recent demonstration in a retrospective cohort study of 446 patients with COVID-19 that early administration of IFN- $\alpha$ (IFN-a2b) is linked to reduced 

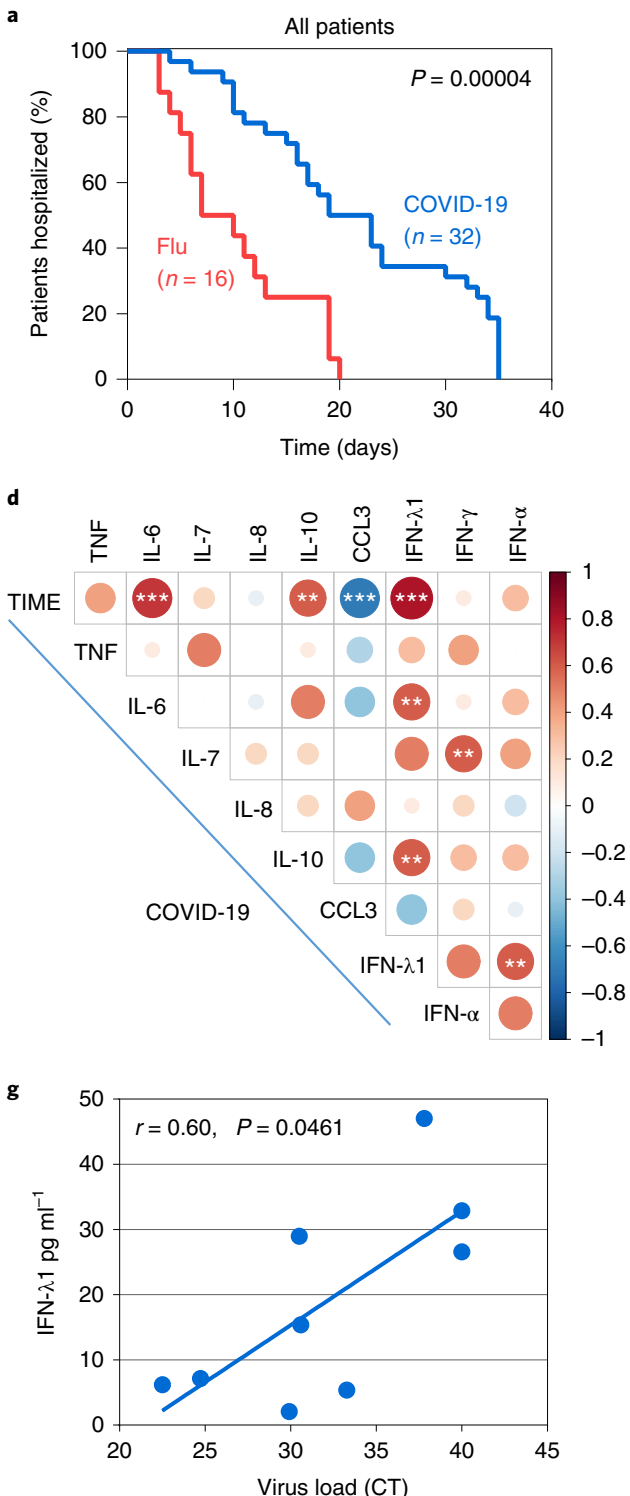
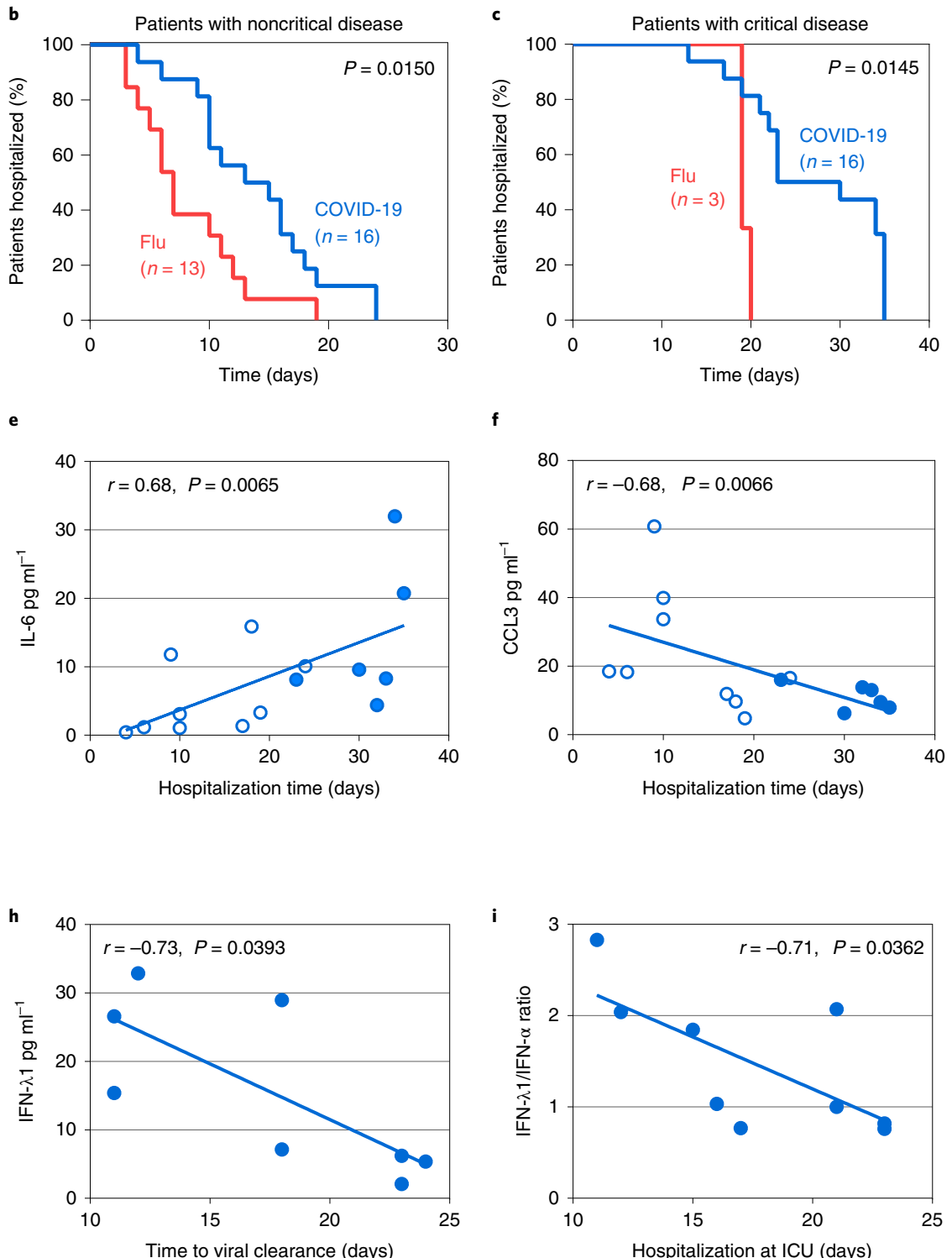

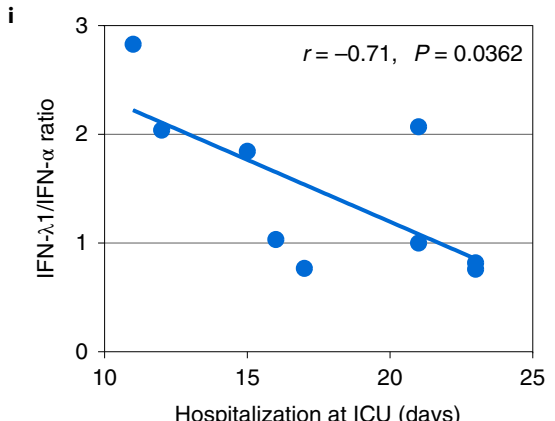

Fig. $\mathbf{5}$ | Correlation of IFN and cytokine expression patterns with disease outcomes. a-c, Comparison of hospitalization time between patients with COVID-19 and flu. All patients (a), patients with noncritical disease (b) and patients with critical disease (c) are shown. d, Correlation matrix of cytokine concentration levels in serum at the day 1-3 time interval after hospital admission of patients with COVID-19, indicating correlations between cytokines and total hospitalization time (TIME) or other cytokines. e,f, Correlation of IL-6 (e) and CCL3 (f) levels in serum with the duration of total hospitalization of all patients with COVID-19. g, Correlation of IFN- $\lambda 1$ levels with viral load expressed as computed tomography values in bronchial aspirates collected at the same time interval as the sera used for IFN- $\lambda 1$ quantification. $\mathbf{h}$, Correlation of IFN- $\lambda 1$ levels with time required for viral clearance assessed as the first negative SARS-CoV-2 test. $\mathbf{i}$, Correlation of IFN- $\lambda$ I:IFN- $\alpha$ ratio with the duration of hospitalization in the ICU. Dots show individual measurements of patients with COVID-19. Open and shaded dots correspond to noncritically and critically ill patients with COVID-19, respectively (e-i). For a-c, $P$ values for the comparison of hospitalization curves between patient groups were determined using the log-rank test. For $\mathbf{d}-\mathbf{i}, P$ values for the strength and direction of association between the two variables, as indicated in each panel, were determined using the Spearman rank-order correlation coefficient for nonparametric data. ${ }^{\star \star} P<0.01$ and ${ }^{\star \star \star} P<0.001$.

in-hospital mortality, whereas late IFN- $\alpha$ therapy leads to increased mortality and delayed recovery leaves little doubt that the timing of IFN production is also crucial in patients with COVID-19 (ref. ${ }^{29}$ ). Conceivably, late production of type I or III IFN production might confer no viral resistance, but instead promote immunopathology.

Whether this unique clinical course of COVID-19 is related to the presence of SARS-CoV-2-derived IFN inhibitors as previously proposed for SARS-CoV ${ }^{30,31}$ and MERS-CoV ${ }^{32}$ is not known but is a possibility. As with other viruses, inhibition may be overcome once higher viral loads are reached, for example after incubation of the virus and eventual spread in susceptible individuals. In our study, we did not see significant differences in virus levels between noncritically and critically ill patients at the time that IFNs were measured (Extended Data Fig. 9). However, higher virus load in severe over mild disease has been described in one study but not been confirmed in another ${ }^{33,34}$. Moreover, higher virus load can overcome SARS-CoV-2 dose-dependent suppression of IFN production in cultured respiratory epithelial cells ${ }^{16}$.

Our study is not without caveats. First, it characterizes cytokine patterns in the circulation and although these are commonly used to analyze 'cytokine storms' in response to infection, how well they correlate to immune responses in the respiratory tract is difficult to 
know. Second, our study is relatively small and our findings await validation in other cohorts. Still, our study is uniquely informative as it addresses the production of IFNs and the activation of the 'cytokine storm' in COVID-19 in a temporal manner, from hospital admission to ICU entry, and should therefore be particularly useful for the design of clinical trials testing IFN therapies. It also describes biomarkers such as IL- 6 and CCL3 and gene expression signatures that can be particularly useful for assessing the risk for critical disease development and duration of hospitalization of patients with COVID-19 who are newly admitted to hospital. Finally, it provides a side-by-side comparison of COVID-19 with flu, studying patient populations with similar genetic, demographic and clinicopathological characteristics, and therefore uncovers important differences in the antiviral immune response between these two diseases that have not been previously suspected.

\section{Online content}

Any methods, additional references, Nature Research reporting summaries, source data, extended data, supplementary information, acknowledgements, peer review information; details of author contributions and competing interests; and statements of data and code availability are available at https://doi.org/10.1038/ s41590-020-00840-x.

Received: 4 June 2020; Accepted: 9 November 2020; Published online: 4 December 2020

\section{References}

1. Sen, G. C. Viruses and interferons. Annu. Rev. Microbiol. 55, 255-281 (2001).

2. McNab, F., Mayer-Barber, K., Sher, A., Wack, A. \& O'Garra, A. Type I interferons in infectious disease. Nat. Rev. Immunol. 15, 87-103 (2015).

3. Guan, W. J. et al. Clinical characteristics of coronavirus disease 2019 in China. N. Engl. J. Med. 382, 1708-1720 (2020).

4. Huang, C. et al. Clinical features of patients infected with 2019 novel coronavirus in Wuhan, China. Lancet 395, 497-506 (2020).

5. Wolfel, R. et al. Virological assessment of hospitalized patients with COVID-2019. Nature 581, 465-469 (2020).

6. Zheng, S. et al. Viral load dynamics and disease severity in patients infected with SARS-CoV-2 in Zhejiang province, China, January-March 2020: retrospective cohort study. Brit. Med. J. 369, m1443 (2020).

7. Taubenberger, J. K. \& Morens, D. M. The pathology of influenza virus infections. Annu. Rev. Pathol. 3, 499-522 (2008).

8. Paules, C. \& Subbarao, K. Influenza. Lancet 390, 697-708 (2017).

9. Lessler, J. et al. Incubation periods of acute respiratory viral infections: a systematic review. Lancet Infect. Dis. 9, 291-300 (2009).

10. Mehta, P. et al. COVID-19: consider cytokine storm syndromes and immunosuppression. Lancet 395, 1033-1034 (2020).

11. Wu, C. et al. Risk factors associated with acute respiratory distress syndrome and death in patients with coronavirus disease 2019 pneumonia in Wuhan, China. JAMA Intern. Med. 180, 934-943 (2020).

12. Yang, $X$. et al. Clinical course and outcomes of critically ill patients with SARS-CoV-2 pneumonia in Wuhan, China: a single-centered, retrospective, observational study. Lancet Respir. Med. 8, 475-481 (2020).
13. Galani, I. E. et al. Interferon- $\lambda$ mediates non-redundant front-line antiviral protection against influenza virus infection without compromising host fitness. Immunity 46, 875-890.e6 (2017).

14. Davidson, S., Crotta, S., McCabe, T. M. \& Wack, A. Pathogenic potential of interferon $\alpha \beta$ in acute influenza infection. Nat. Commun. 5, 3864 (2014).

15. Andreakos, E., Zanoni, I. \& Galani, I. E. $\lambda$ interferons come to light: dual function cytokines mediating antiviral immunity and damage control. Curr. Opin. Immunol. 56, 67-75 (2019).

16. Blanco-Melo, D. et al. Imbalanced host response to SARS-CoV-2 drives development of COVID-19. Cell 181, 1036-1045 (2020).

17. Hadjadj, J. et al. Impaired type I interferon activity and inflammatory responses in severe COVID-19 patients. Science 369, 718-724 (2020).

18. World Health Organization. Clinical management of COVID-19: interim guidance, 27 May 2020. https://apps.who.int/iris/handle/10665/332196 (2020).

19. Lim, W. S. et al. Defining community acquired pneumonia severity on presentation to hospital: an international derivation and validation study. Thorax 58, 377-382 (2003).

20. Rathinam, V. A. \& Fitzgerald, K. A. Inflammasome complexes: emerging mechanisms and effector functions. Cell 165, 792-800 (2016).

21. Galani, I. E. \& Andreakos, E. Neutrophils in viral infections: current concepts and caveats. J. Leukoc. Biol. 98, 557-564 (2015).

22. Mangalmurti, N. \& Hunter, C. A. Cytokine storms: understanding COVID-19. Immunity 53, 19-25 (2020).

23. Chen, G. et al. Clinical and immunological features of severe and moderate coronavirus disease 2019. J. Clin. Invest. 130, 2620-2629 (2020).

24. Liu, T. et al. The role of interleukin-6 in monitoring severe case of coronavirus disease 2019. EMBO Mol. Med. 12, e12421 (2020).

25. Channappanavar, R. et al. Dysregulated type I interferon and inflammatory monocyte-macrophage responses cause lethal pneumonia in SARS-CoV-infected mice. Cell Host Microbe 19, 181-193 (2016).

26. Broggi, A. et al. Type III interferons disrupt the lung epithelial barrier upon viral recognition. Science 369, 706-712 (2020).

27. Major, J. et al. Type I and III interferons disrupt lung epithelial repair during recovery from viral infection. Science 369, $712-717$ (2020).

28. Wang, B. X. \& Fish, E. N. The yin and yang of viruses and interferons. Trends Immunol. 33, 190-197 (2012).

29. Wang, N. et al. Retrospective multicenter cohort study shows early interferon therapy is associated with favorable clinical responses in COVID-19 patients. Cell Host Microbe 28, 455-464 (2020).

30. Wathelet, M. G., Orr, M., Frieman, M. B. \& Baric, R. S. Severe acute respiratory syndrome coronavirus evades antiviral signaling: role of nspl and rational design of an attenuated strain. J. Virol. 81, 11620-11633 (2007).

31. Kopecky-Bromberg, S. A., Martínez-Sobrido, L., Frieman, M., Baric, R. A. \& Palese, $\mathrm{P}$. Severe acute respiratory syndrome coronavirus open reading frame (ORF) 3b, ORF 6, and nucleocapsid proteins function as interferon antagonists. J. Virol. 81, 548-557 (2007).

32. Comar, C. E. et al. Antagonism of dsRNA-induced innate immune pathways by NS4a and NS4b accessory proteins during MERS coronavirus infection. Mbio 10, e00319 (2019).

33. Liu, Y. et al. Viral dynamics in mild and severe cases of COVID-19. Lancet Infect. Dis. 20, 656-657 (2020).

34. He, X. et al. Temporal dynamics in viral shedding and transmissibility of COVID-19. Nat. Med. 26, 672-675 (2020).

Publisher's note Springer Nature remains neutral with regard to jurisdictional claims in published maps and institutional affiliations.

(c) The Author(s), under exclusive licence to Springer Nature America, Inc. 2020 


\section{Methods}

Study participants. In this noninterventional study, 32 patients with a diagnosis of COVID-19 pneumonia according to World Health Organization guidelines and positive SARS-CoV-2 PCR with reverse transcription (RT-PCR) testing on a respiratory sample (nasopharyngeal swab or bronchial aspirate) were included ${ }^{18}$. Patients were recruited between March and April 2020 from the $1^{\text {st }}$ Respiratory and Critical Care Clinic ward and ICU of the 'Sotiria' General Chest Diseases Hospital of Athens, Greece. Healthy, asymptomatic individuals with a negative SARS-CoV-2 RT-PCR at the time of inclusion served as the control group.

The severity of COVID-19 cases was classified based on the adaptation of the Seventh Revised Trial Version of the Novel Coronavirus Pneumonia Diagnosis and Treatment Guidance ${ }^{35}$. All patients had moderate-to-severe disease and presented with respiratory symptoms and radiological findings of pneumonia. They met any of the following criteria:

1. Respiratory distress $\left(\geq 30\right.$ breaths $\left.\mathrm{min}^{-1}\right)$;

2. Oxygen saturation $\leq 93 \%$ at rest;

3. Arterial partial pressure of oxygen $\left(\mathrm{PaO}_{2}\right) /$ fraction of inspired oxygen $\left(\mathrm{FiO}_{2}\right)$ $\leq 300 \mathrm{~mm} \mathrm{Hg}$ with no other organ failure.

Sixteen patients developed ARDS and critical illness due to respiratory failure $\left(\mathrm{PaO}_{2} / \mathrm{FiO}_{2} \leq 200 \mathrm{~mm} \mathrm{Hg}\right)$ requiring mechanical ventilation, with shock and/ or other organ failure necessitating ICU care. Blood was drawn at various time intervals during hospitalization and at discharge; and WBCs and serum were collected and stored until further use.

To better understand the immune response of COVID-19 infection, individuals from a cohort of patients with confirmed $\mathrm{H} 1 \mathrm{~N} 1 / \mathrm{H} 3 \mathrm{~N} 2$ influenza A virus infection were also studied. In total, 40 patients were recruited between December 2018 and May 2019 at the $2^{\text {nd }}$ Respiratory Clinic of the 'Sotiria' General Chest Diseases Hospital, Athens, Greece and the 'Attikon' University Hospital, University of Athens Medical School, Athens, Greece. Confirmation was obtained from nasopharyngeal swabs using the BioFire FilmArray Respiratory Panel test (bioMerieux). Similarly to patients with COVID-19, those with flu were categorized according to the severity of the disease into mild cases with no radiological findings of pneumonia, no need for oxygen support and hospitalization, and moderate-to-severe cases with radiological findings of pneumonia (X-ray or computed tomography), oxygen need and symptoms requiring hospitalization ${ }^{36}$. Hospitalized patients with flu were subdivided into patients who did not develop $\left(\mathrm{PaO}_{2} / \mathrm{FiO}_{2}>200 \mathrm{~mm} \mathrm{Hg}\right)$ or developed critical disease $\left(\mathrm{PaO}_{2} / \mathrm{FiO}_{2} \leq 200 \mathrm{~mm} \mathrm{Hg}\right)$. Patients with flu had similar clinicopathological characteristics to patients with COVID-19 upon admission (Supplementary Table 1). All those included in the study were clinically evaluated and followed longitudinally during the whole period of hospitalization (from admission to discharge). All blood specimens were processed immediately for serum collection and aliquots were stored at $-80^{\circ} \mathrm{C}$. Additionally, serum from ten healthy individuals, three male and seven female, with a median age of 54.5 (41-70) years, was also collected and used as a control group.

The study conforms to the principles outlined in the Declaration of Helsinki and received approval by the Ethics Committees of the 'Sotiria' General Chest Diseases Hospital, Athens, Greece (approval numbers 16707/10-7-18 and 8385/313-20) and the 'Attikon' University Hospital, University of Athens Medical School, Athens, Greece (approval number 1821A/22-9-16). All participants provided written informed consent.

SARS-CoV-2 detection. RNA was extracted from nasopharyngeal swabs and bronchial aspirates by using the Nuclisens easyMAG instrument (bioMerieux) according to the manufacturer's instructions. Nasopharyngeal swabs were used for initial diagnosis, while bronchial aspirates were used for assessing viral load in the ICU. Real time RT-PCR was performed on extracted nucleic acids targeting the E gene of SARS-CoV-2 as described previously ${ }^{37}$.

Cytokine analysis. Serum samples frozen and stored at $-20^{\circ} \mathrm{C}$, without other thawing, were analyzed for the presence of IFN- $\gamma$, TNF, IL- $1 \beta$, IL-2, IL- 4 , IL-6, IL-7, IL-8, IL-10, IL-12 (p70), IL-13, IL-17A, IL-23, CCL3, CCL4 and CX3CL1 with the MILLIPLEX MAP Human High-Sensitivity T cell Panel (Merck Millipore). Thawed serum aliquots were centrifuged at 13,000 r.p.m. for $10 \mathrm{~min}$ at $4{ }^{\circ} \mathrm{C}$ immediately before testing. Each assay was performed according to the manufacturer's protocol for serum samples, utilizing recommended sample dilutions and standard curve concentrations (Merck Millipore). Samples were analyzed on a Luminex 200 System using Luminex xPonent v.3.1 software according to the manufacturer's instructions (Merck Millipore). For each cytokine on each assay, the lowest detection limits were in $\mathrm{pg} \mathrm{ml}^{-1}: 0.50$ for IFN- $\gamma, 0.42$ for TNF, 0.2 for IL-1 $\beta, 0.24$ for IL-2, 0.60 for IL-4, 0.16 for IL-6, 0.33 for IL-7, 0.30 for IL-8, 0.50 for IL-10, 0.24 for IL-12 (p70), 0.20 for IL-13, 0.50 for IL-17A, 8.00 for IL-23, 2.00 for CCL3, 0.80 for CCL4 and 10.00 for CX3CL1. High-sensitivity sandwich ELISA kits were used for the detection of human IFN- $\alpha$ (Abcam) and IFN- $\lambda 1$ (BioLegend). Their sensitivity in $\mathrm{pg} \mathrm{ml}^{-1}$ was 1.00 for IFN- $\alpha$ and 2.00 for IFN- $\lambda 1$. Data were acquired and analyzed using Biotek Gen 5 v.1.05 software.

RNA-seq analysis. For RNA-seq analysis, total RNA was purified from whole blood leukocytes with the RNeasy Micro kit (QIAGEN). RNA samples were treated with DNase I (QIAGEN) and quantified on a NanoDrop (Thermo Fisher Scientific). Next-generation sequencing libraries were prepared with the TruSeq RNA Library Prep kit v.2 (Illumina) according to the manufacturer's instructions. Quality of the libraries was validated with an Agilent DNA 1000 kit run on an Agilent 2100 Bioanalyzer. This analysis returned the $\log _{2}$ fold changes of the treatment compared to healthy individuals for each time point. DEG transcripts of all, critically ill and noncritically ill patients with COVID-19 were selected based on an adjusted $P$ value cutoff of 0.05 (false discovery rate (FDR) of 5\%). Additional statistical comparison of critically ill and noncritically ill COVID-19 DEGs was performed. Pathway-enrichment analysis was conducted on DEGs over those of healthy individuals using the ClueGO and CluePedia plugin of Cytoscape. Heat maps were performed using TM4 MeV v.4.8 and Euclidean distance was used for hierarchical clustering. Clustering and dendrograms were performed with the hclust function and ggdendro package, respectively, in $\mathrm{R}$.

Transcriptomics analyses. Samples sequenced on NextSeq 500 (Illumina) were analyzed using standard protocols. Briefly, raw reads were pre-processed using FastQC v.0.11.2 and cutadapt v.1.6, and then mapped to the human genome (GRCh38) using the TopHat version 2.0.13, Bowtie v.1.1.1 and Samtools version v.1.1. The read count table was produced using HTSeq v.0.6. Following filtering of raw read counts with a threshold of 10 in at least one dataset, resulting in a total of 21,880 genes. DESeq 2 analysis ${ }^{38}$ was performed among the critically ill and noncritically ill patients and healthy individuals, as well as among the critically ill and noncritically ill patients. This analysis returned the $\log _{2}$ fold changes of the treatment compared to healthy individuals for each time point. DEG transcripts were selected based on an adjusted $P$ value cutoff of 0.05 (FDR 5\%). Pathway-enrichment analysis was conducted using the ClueGO and CluePedia plugin of Cytoscape. Heat maps were performed using TM4 MeV v.4.8 and Euclidean distance was used for hierarchical clustering. Clustering and dendrograms were performed with the hclust function and ggdendro package, respectively, in $\mathrm{R}$.

Statistical analysis. Data were analyzed on GraphPad Prism software. Statistical significance of differences among cytokine levels was assessed using the Mann-Whitney $U$-test for nonparametric data. Associations between cytokine levels and hospitalization time (in days) were tested using Spearman rank-order correlation coefficient and visualized using the corrplot R package. Statistical significance of differences among gene groups was assessed using a two-tailed Student's $t$-test for parametric data. Polar charts from the ggplot $2 \mathrm{R}$ package were used for the visualization of differences in cytokine response patterns.

Reporting Summary. Further information on research design is available in the Nature Research Reporting Summary linked to this article.

\section{Data availability}

The data that support the findings of this study are available from the corresponding author upon request. The raw RNA-seq data have been deposited at Gene Expression Omnibus (http://www.ncbi.nlm.nih.gov/geo/) under BioProject accession number PRJNA638753 and are publicly available.

\section{References}

35. National Health Commission \& National Administration of Traditional Chinese Medicine. Diagnosis and treatment protocol for novel coronavirus pneumonia (trial version 7). Chin. Med. J. (Engl.) 133, 1087-1095 (2020).

36. Uyeki, T. M. et al. Clinical practice guidelines by the Infectious Diseases Society of America: 2018 update on diagnosis, treatment, chemoprophylaxis, and institutional outbreak management of seasonal influenza. Clin. Infect. Dis. 68, 895-902 (2019).

37. Corman, V. M. et al. Detection of 2019 novel coronavirus (2019-nCoV) by real-time RT-PCR. Eur. Surveill. 25, 2000045 (2020).

38. Love, M. I., Huber, W. \& Anders, S. Moderated estimation of fold change and dispersion for RNA-seq data with DESeq2. Genome Biol. 15, 550 (2014).

\section{Acknowledgements}

The authors thank A. Gavriil and G. Vatsellas for technical assistance and P. Katsikis (Erasmus Medical Center, the Netherlands) and V. Soumelis (Institut de Recherche Saint-Louis, Université de Paris, France) for critically reading the manuscript. E.A. is supported by research grants from the European Commission (IMMUNAID, no. 779295 and CURE, no. 767015), the Hellenic Foundation for Research and Innovation (INTERFLU, no. 1574) and Janssen Pharmaceuticals. I.E.G. is supported by a research grant from the Hellenic Foundation for Research and Innovation (RELIEVE, no. 506). This work was supported by a grant from the Hellenic Thoracic Society and a donation from Jorge Andres Sanchez Papaspiliou family and friends. 


\section{Author contributions}

N.R., S.T., A.K. and E.A. designed the study; I.E.G., N.R., V.L., V.T., E.K., P.C.F., V.P., V.R., O.K. and E.A. conducted patient data and sample collection; V.L. and E.A. processed and prepared the samples for downstream analyses; E.K., P.C.F., V.P., V.R., O.K. and N.K. conducted medical evaluation; I.E.G., N.R., V.L., V.T. and E.A. performed cytokine measurements; I.E.G., M.M. and E.A. performed RNA-seq analyses; A.M. measured viral load; I.E.G., N.R., V.L., V.T., M.M., E.P., E.K., A.K. and E.A. analyzed and interpreted the data; E.A. drafted the manuscript and supervised the study.

\section{Competing interests}

The authors declare no competing interests.

\section{Additional information}

Extended data is available for this paper at https://doi.org/10.1038/s41590-020-00840-x. Supplementary information is available for this paper at https://doi.org/10.1038/ s41590-020-00840-x.

Correspondence and requests for materials should be addressed to E.A.

Peer review information Peer reviewer reports are available. Nature Immunology thanks the anonymous reviewers for their contribution to the peer review of this work. L. A Dempsey was the primary editor on this article and managed its editorial process and peer review in collaboration with the rest of the editorial team.

Reprints and permissions information is available at www.nature.com/reprints. 
a.

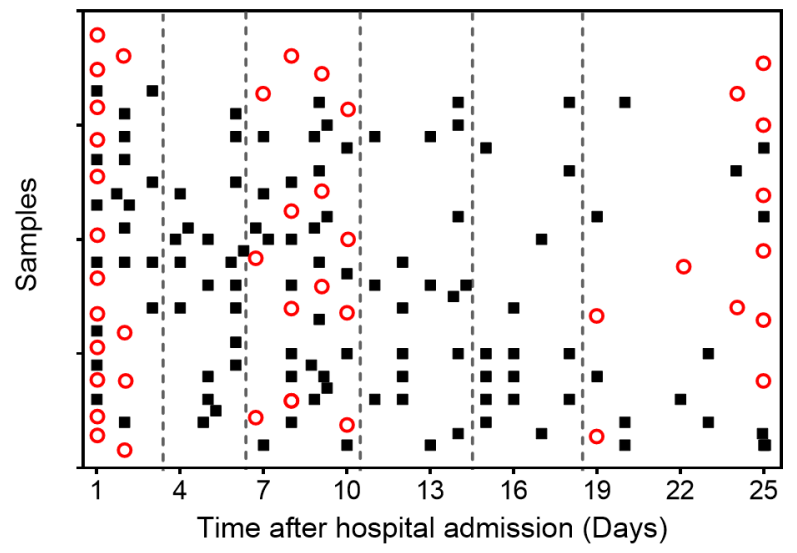

b.

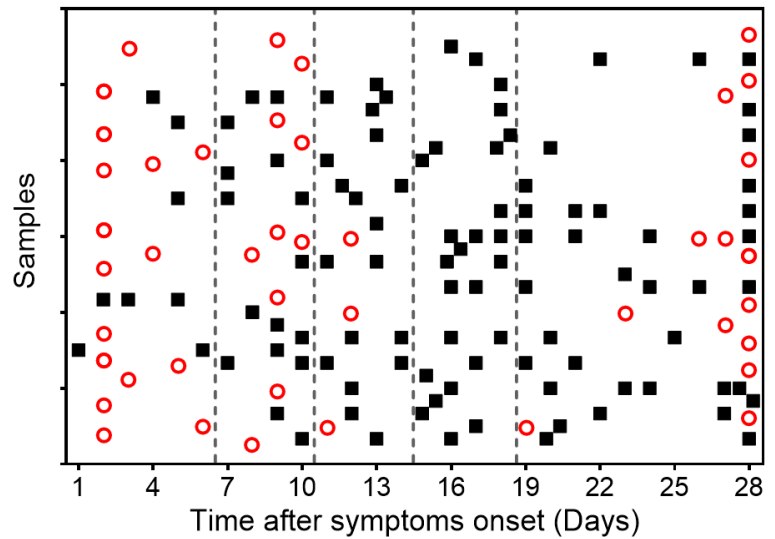

Extended Data Fig. 1 | Distribution of COVID-19 and flu patient samples analyzed in the study. a, Samples ordered in relation to hospital admission. b. Samples ordered in relation to disease onset. Black squares represent individual samples from 32 COVID-19 patients and red dots from 16 flu patients hospitalized for pneumonia. 

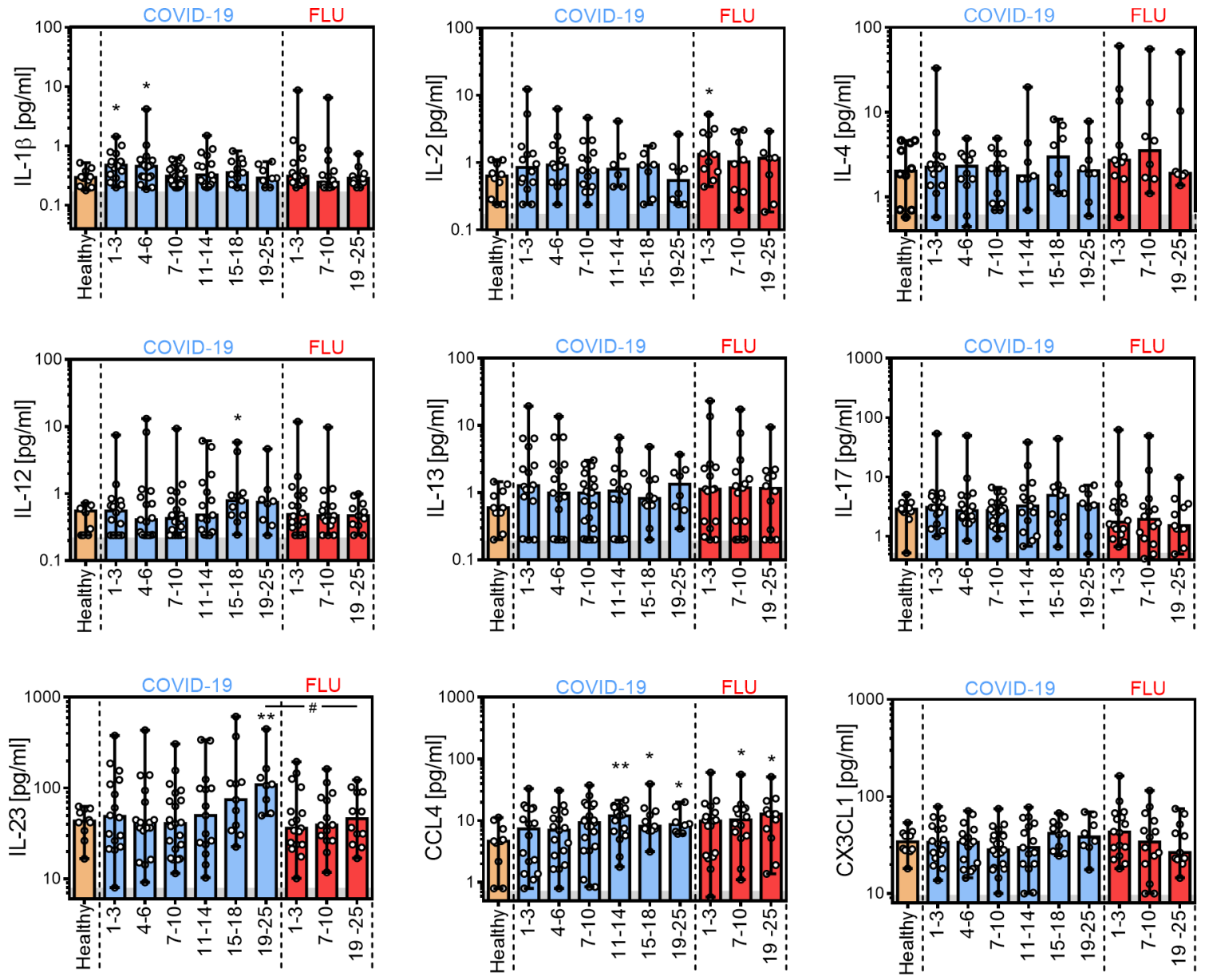

Extended Data Fig. 2 | Temporal cytokine patterns of COVID-19 and flu patients in relation to hospital admission. Levels of IL-13, IL-2, IL-4, IL-12, IL-13, IL-17, IL-23, CCL4 and CX3CL1 concentration at various time intervals after hospital admission of 32 COVID-19 and 16 flu patients with pneumonia followed longitudinally, and 10 healthy individuals. Data are presented as scatter plots with dots showing individual patient measurements, columns median values and error bars range. For COVID-19, $n=16,17,21,15,11$ and 8 for each of the six consecutive time intervals. For flu, $n=16,14$ and 11 , respectively. For healthy, $\mathrm{n}=10$. Grey shading marks the limit of quantification of the assay. $P$ values were determined by a two tailed Mann-Whitney $U$ test for non-parametric comparisons. ${ }^{\star} P<0.05$ and ${ }^{\star \star} P<0.01$ show significance over healthy controls. ${ }^{\sharp} P<0.05$ shows significance between COVID-19 and flu groups. 
a.

\begin{tabular}{|c|c|c|c|c|c|c|c|}
\hline Symptoms onset & & & Samp & ng inter & als & & \\
\hline & $1-6$ & $7-10$ & $11-14$ & $15-18$ & $15-18$ & $19-28$ & Id) \\
\hline
\end{tabular}

b.
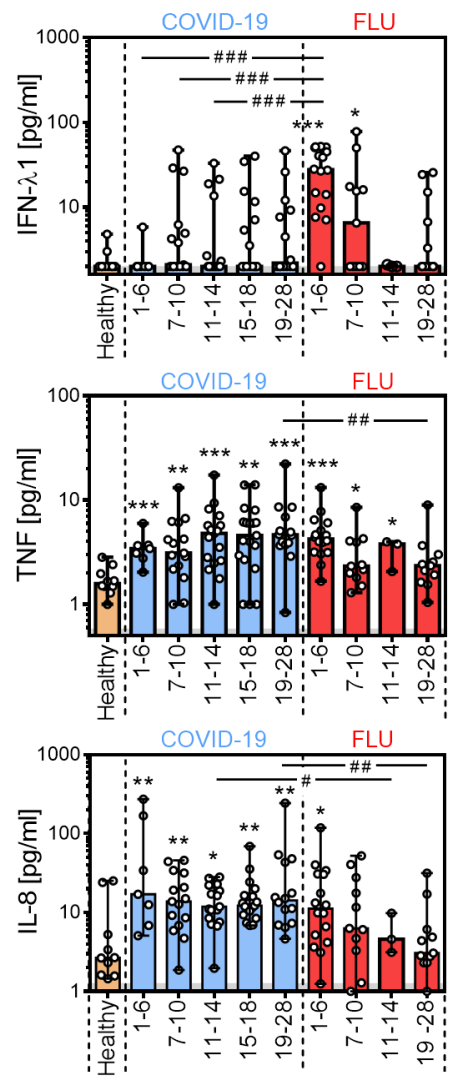

C.
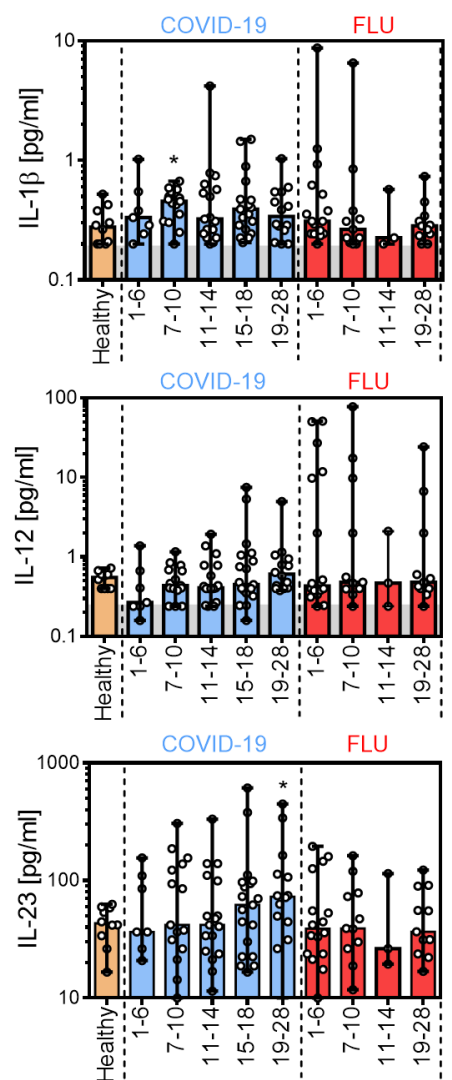
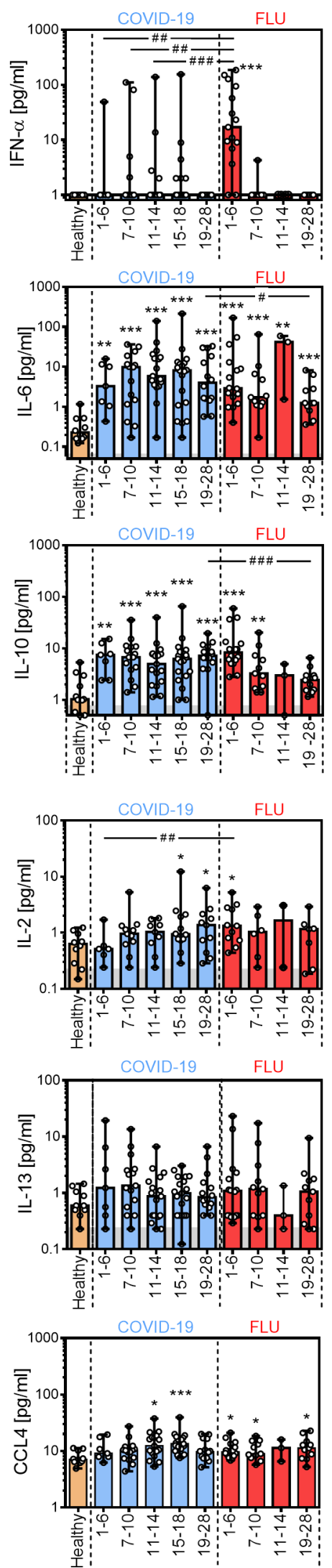
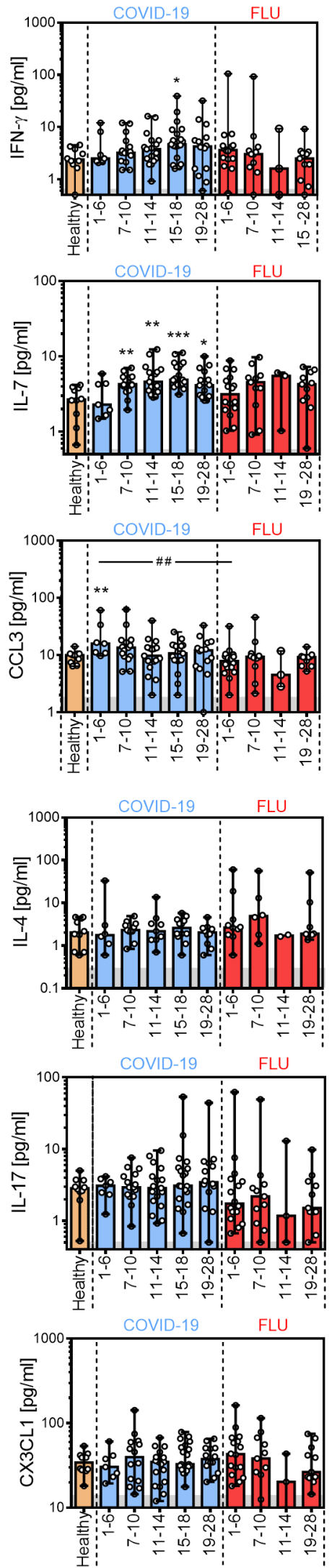
Extended Data Fig. 3 | Temporal cytokine patterns of COVID-19 and flu patients in relation to disease symptoms onset. a, Schematic showing the experimental design with sampling at specific time intervals after disease symptoms onset of 32 COVID-19 and 16 flu patients with pneumonia, and 10 healthy individuals. b, Levels of IFN- $\lambda 1$, IFN- $\alpha$, IFN- $\gamma$, TNF, IL- 6, IL-7, IL-8, IL-10 and CCL3 concentration at various time intervals after disease symptoms onset. c, Levels of IL-1 1 , IL-2, IL-4, IL-12, IL-13, IL-17, IL-23, CCL4 and CX3CL1 concentration at various time intervals after disease symptoms onset. Data are presented as scatter plots with dots showing individual patient measurements, columns median values and error bars range. For COVID-19, $\mathrm{n}=7,15$, 17,19 and 14 for each of the five consecutive time intervals. For flu, $n=16,11,3$ and 11, respectively. For healthy, $n=10$. Grey shading marks the limit of quantification of the assay. $P$ values were determined by a two tailed Mann-Whitney $U$ test for non-parametric comparisons. ${ }^{\star} P<0.05$, ${ }^{\star \star} P<0.01$ and ${ }^{* \star *} P<0.001$ show significance over healthy controls. ${ }^{\#} P<0.05,{ }^{\# \# P}<0.01$ and ${ }^{\# \#} P<0.001$ show significance between COVID-19 and flu groups. 
a.

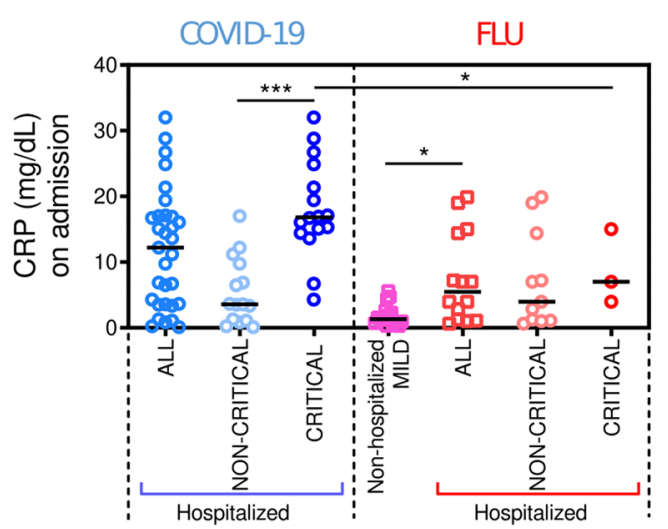

C.

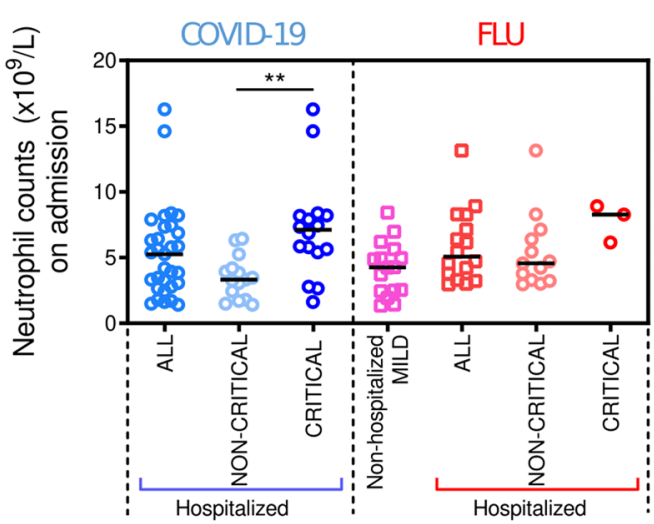

e.

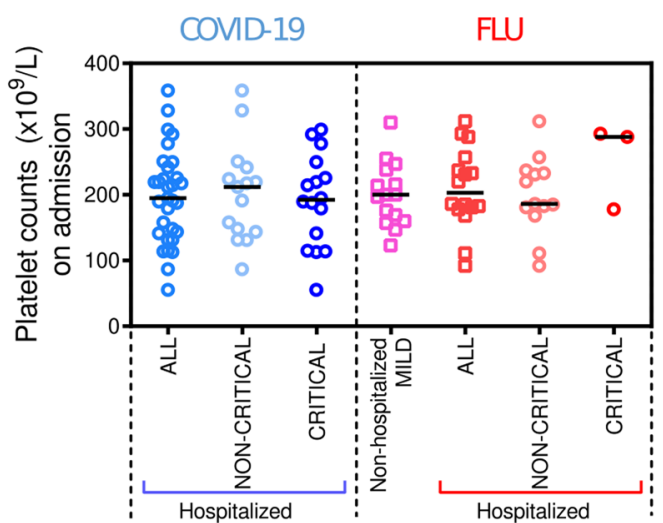

g.

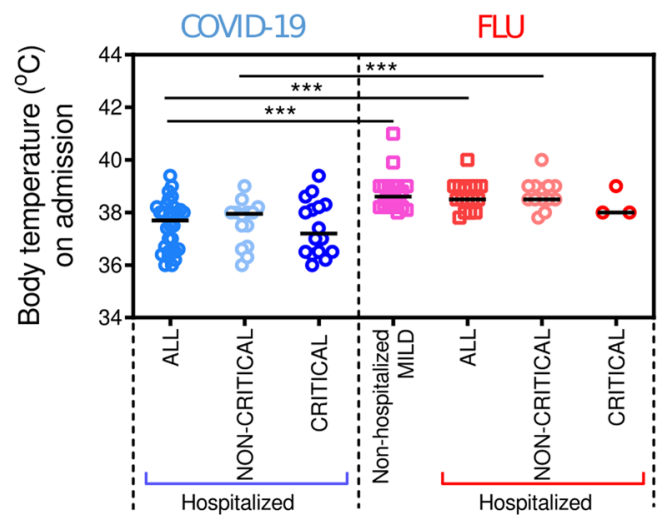

b.

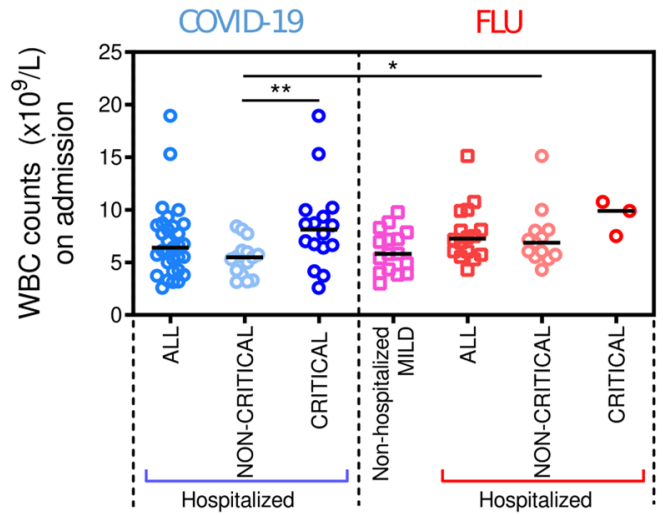

d.

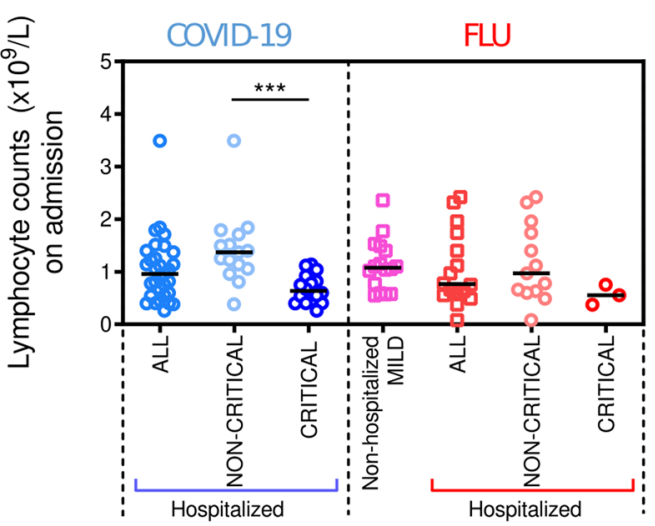

f.

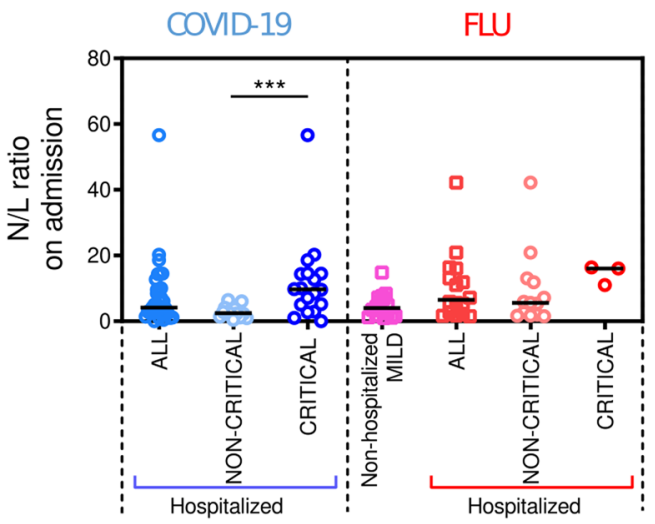

h.

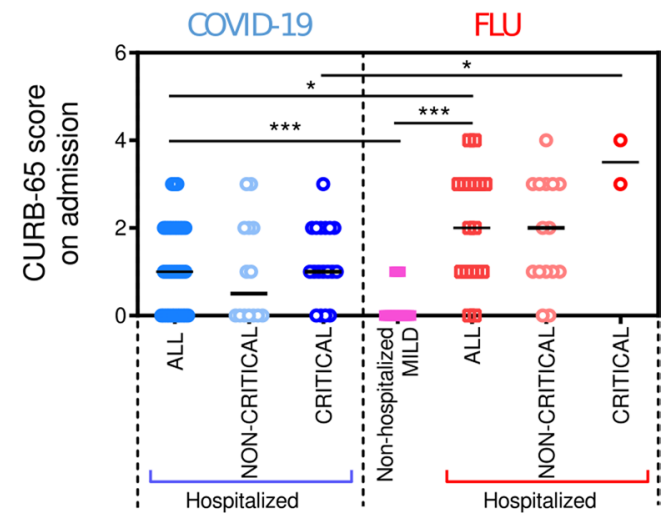

Extended Data Fig. 4 | See next page for caption. 
Extended Data Fig. 4 | Clinical laboratory parameters of COVID-19 and flu patients on admission. 32 COVID-19 and 16 flu patients hospitalized for pneumonia, and 24 non-hospitalized flu patients with no radiological findings of pneumonia were analyzed. Subgroups of hospitalized patients developing critical versus non-critical disease were also assessed. $\mathbf{a}$, Serum CRP levels of the various patient groups. $\mathbf{b}$-e, White blood cell (WBC) (b), neutrophil (c), lymphocyte (d) and platelet (e) counts per liter in peripheral blood. $\mathbf{f}$, Neutrophil to lymphocyte (N/L) ratio in peripheral blood. $\mathbf{g}$, Body temperature of the various patient groups measured in ${ }^{\circ} \mathrm{C}$. $\mathbf{h}$, CURB- 65 score indicative of pneumonia severity of the various patient groups on hospital admission or visit. Data are presented as scatter plots with dots showing individual patient measurements and lines median values. For COVID-19, $n=32$ hospitalized patients of which 16 were non-critically and 16 critically ill. For flu, $n=16$ hospitalized patients of which 13 were non-critically and 3 critically ill, as well as 24 mild non-hospitalized patients. $P$ values were determined by a two tailed Mann-Whitney U test for non-parametric comparisons. ${ }^{\star} P<0.05,{ }^{\star \star} P<0.01$ and ${ }^{* \star *} P<0.001$ show significance over healthy controls. 


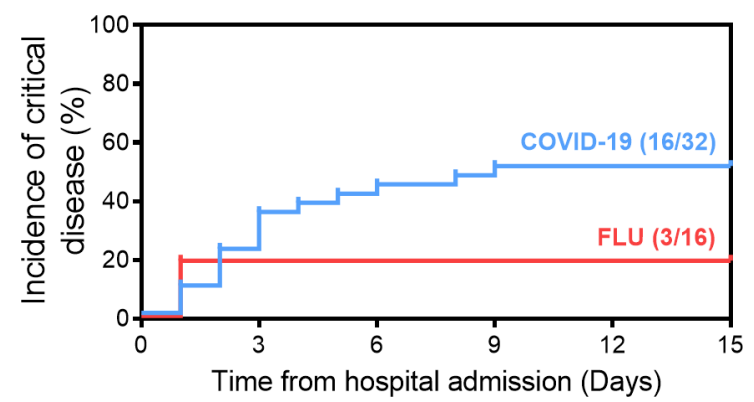

Extended Data Fig. 5 | Incidence of critical disease in hospitalized COVID-19 and flu patients over time. 32 COVID-19 and 16 flu patients hospitalized for pneumonia were followed longitudinally and development of critical disease noted. In total, 50\% (16/32) of COVID-19 patients and 19\% (3/16) of flu patients became critically ill. 

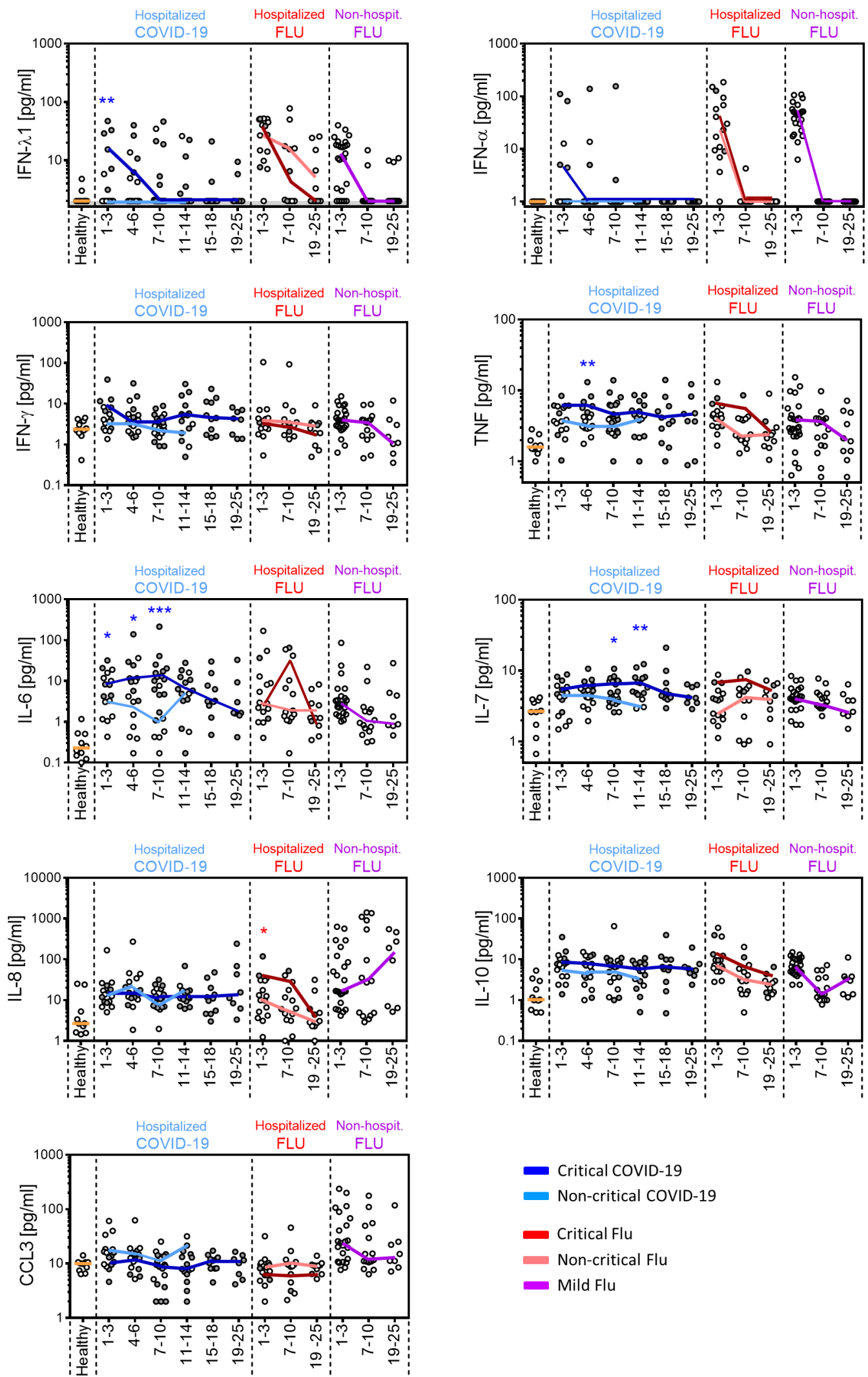

$$
\begin{aligned}
& \text { Critical COVID-19 } \\
& \text { Non-critical COVID-19 } \\
& \text { Critical Flu } \\
& \text { Non-critical Flu } \\
& \text { Mild Flu }
\end{aligned}
$$

Extended Data Fig. 6 | See next page for caption. 


\section{NATURE IMMUNOLOGY}

Extended Data Fig. 6 | Comparison of temporal cytokine patterns among subgroups of COVID-19 and flu patients developing critical versus non-critical disease. Temporal expression of IFN- $\lambda 1$, IFN- $\alpha$, IFN- $\gamma$, TNF, IL-6, IL-7, IL-8, IL-10 and CCL3 of critically or non-critically ill hospitalized patients, and mild non-hospitalized patients after hospital admission or visit, respectively, as well as healthy individuals. Colored lines represent median values of each subgroup over time. Dots show individual patient measurements and shaded dot plots values corresponding to critically ill patients. For COVID-19, $n=16,17,21,15,11$ and 8 for each of the six consecutive time intervals. For hospitalized flu, $n=16,14$ and 11, and for mild non-hospitalized flu, $n=24$, 15 and 14 , for each of the three consecutive time intervals respectively. For healthy, $n=10$. Grey shading marks the limit of quantification of the assay. $P$ values were determined by a two tailed Mann-Whitney $U$ test for non-parametric comparisons. ${ }^{\star} P<0.05,{ }^{\star \star} P<0.01$ and ${ }^{\star \star \star} P<0.001$ show significance between critically and non-critically ill patients at the specific time interval and patient group. For IFN $\lambda 1$ at the day $1-3$ time interval of COVID-19 patients, $p=0.0017$; For TNF at the days 4-6 time interval of COVID-19 patients $p=0.0046$; For IL-6 at the days 1-3, 4-6 and 7-10 time intervals of COVID-19 patients $p=0.0418,0.0431,0.000022$, respectively. For IL-7 at the days $7-10$ and $11-14$ time intervals of COVID-19 patients $p=0.0299$ and 0.0044 , respectively. For IL-8 at the day $1-3$ time interval of Flu patients $p=0.0484$. 

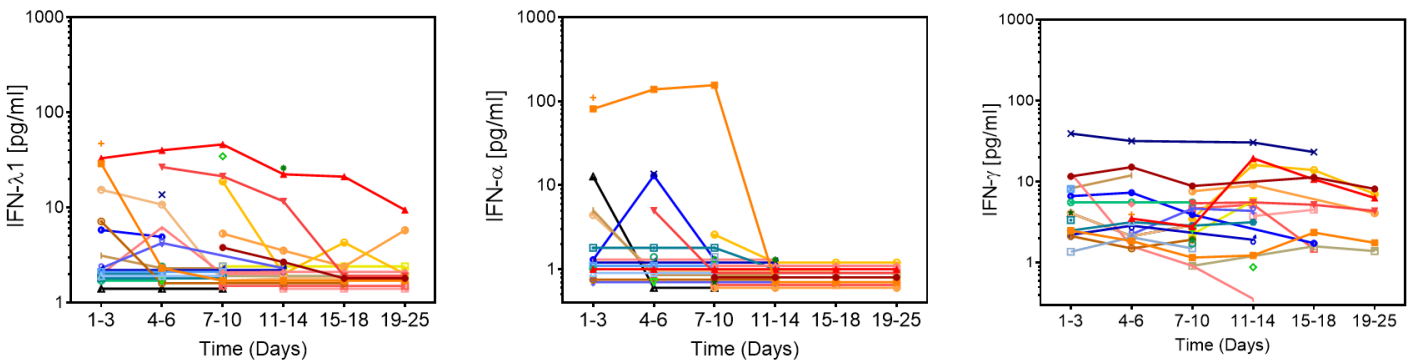

CRITICALLY

$$
\text { ILL }
$$

- Patient 1

- Patient 2

- Patient 3

- Patient 4

- Patient 5

- Patient 13

- Patient 15

- Patient 22

+ Patient 23

$\rightarrow$ Patient 25
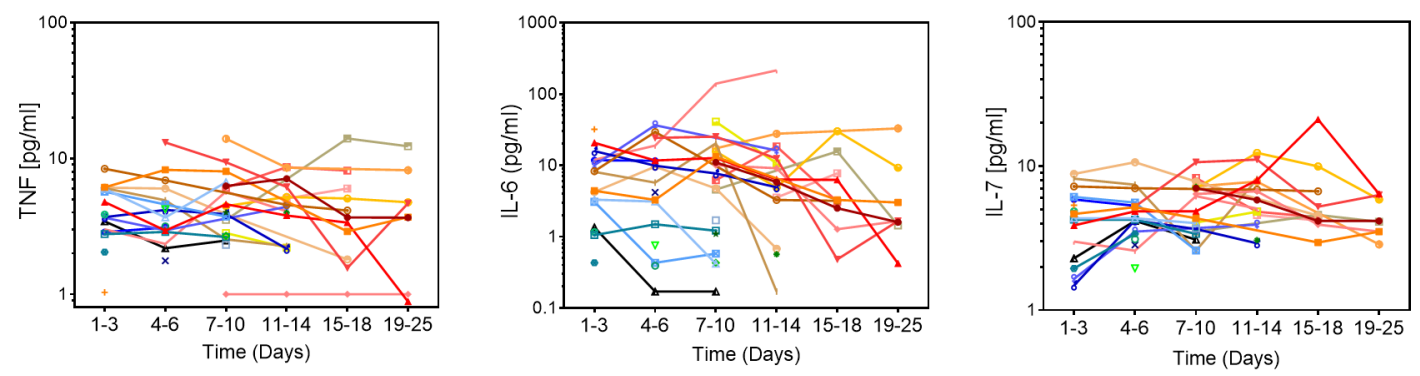

Patient 27

- Patient 28

- Patient 29

- Patient 30

* Patient 31

* Patient 32

- Patient 6

- Patient 7

- Patient 8

- Patient 9

- Patient 10

- Patient 14

- Patient 11

- Patient 12

* Patient 16

- Patient 17
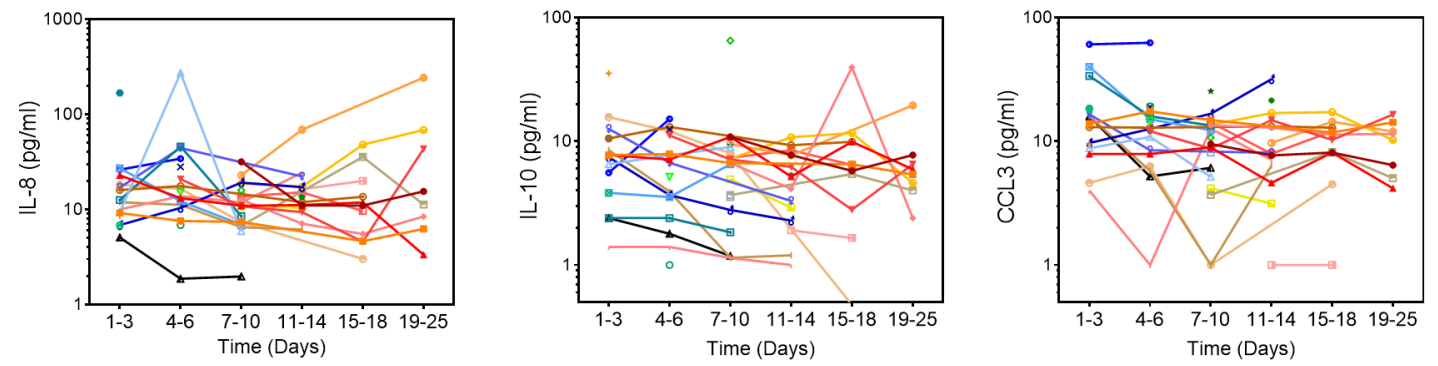

- Patient 19

- Patient 20

+ Patient 21

- Patient 24

- Patient 26

NON-CRITICALLY

ILL

Extended Data Fig. 7 | Temporal cytokine patterns of individual COVID-19 patients. Expression patterns of IFN- $\lambda 1$, IFN- $\alpha$, IFN- $\gamma$, TNF, IL-6, IL-7, IL-8, IL-10 and CCL3 concentration in the sera of 32 individual COVID-19 patients following hospital admission. Distinct color symbols and lines represent individual patient values over time. Patients developing critical versus non-critical disease are shown. 

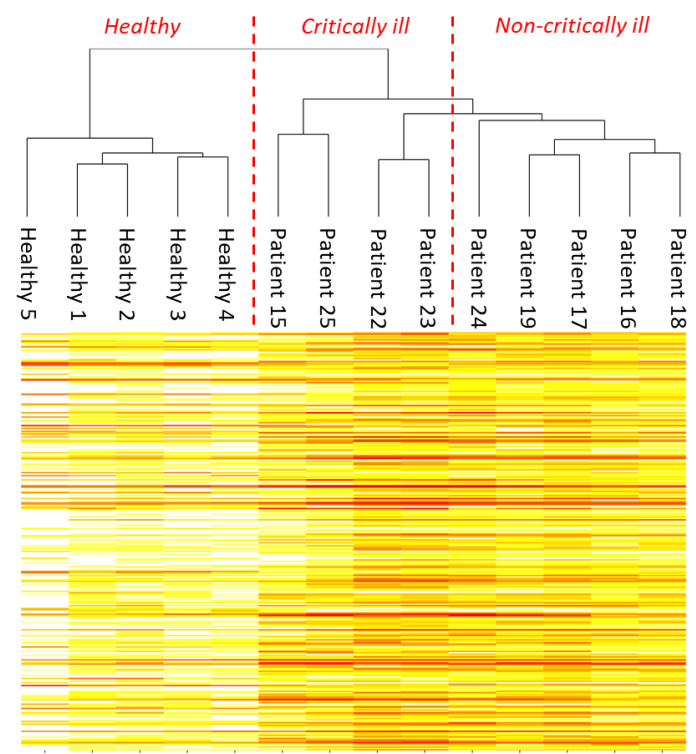

Extended Data Fig. 8 | Clustering of blood transcriptional signatures of COVID-19 patients according to disease severity. Heatmap and hierarchical clustering of the variance stabilizing transformed values for healthy, critically ill and non-critically ill patients at day 1 post-hospitalization based on the genes with a log2-fold-change of at least 3 and adjusted p-value of less than 0.001 between patients and healthy. Hierarchical clustering was done using the unweighted pair group method with arithmetic mean (UPGMA) method provided by the hclust $R$ function, and dendrogram was done using ggdendro R package. 


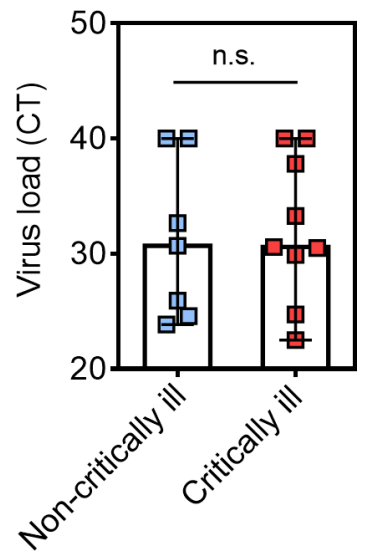

Extended Data Fig. 9 | Comparison of viral load between COVID-19 patients that develop critical versus non-critical disease. Viral load at the day 1-3 time interval of hospital admission of COVID-19 patients that eventually develop critical versus non-critical disease. Data are presented as scatter plots with dots showing individual patient measurements, columns median values and error bars range. $N=8$ for non-critically ill and $n=9$ for critically ill COVID-19 patients. Statistical significance between the two groups was determined by a two tailed Mann-Whitney U test for non-parametric comparisons. n.s.: Not significant. 


\section{Reporting Summary}

Nature Research wishes to improve the reproducibility of the work that we publish. This form provides structure for consistency and transparency in reporting. For further information on Nature Research policies, see our Editorial Policies and the Editorial Policy Checklist.

\section{Statistics}

For all statistical analyses, confirm that the following items are present in the figure legend, table legend, main text, or Methods section.

$\mathrm{n} / \mathrm{a}$ Confirmed

$\bigotimes$ The exact sample size $(n)$ for each experimental group/condition, given as a discrete number and unit of measurement

\ A statement on whether measurements were taken from distinct samples or whether the same sample was measured repeatedly

The statistical test(s) used AND whether they are one- or two-sided

Only common tests should be described solely by name; describe more complex techniques in the Methods section.

Х A description of all covariates tested

Х A description of any assumptions or corrections, such as tests of normality and adjustment for multiple comparisons

$\triangle$ A full description of the statistical parameters including central tendency (e.g. means) or other basic estimates (e.g. regression coefficient)

AND variation (e.g. standard deviation) or associated estimates of uncertainty (e.g. confidence intervals)

For null hypothesis testing, the test statistic (e.g. $F, t, r$ ) with confidence intervals, effect sizes, degrees of freedom and $P$ value noted Give $P$ values as exact values whenever suitable.

Х $\square$ For Bayesian analysis, information on the choice of priors and Markov chain Monte Carlo settings

$\square$ \ For hierarchical and complex designs, identification of the appropriate level for tests and full reporting of outcomes

$\square \bigotimes$ Estimates of effect sizes (e.g. Cohen's d, Pearson's $r$ ), indicating how they were calculated

Our web collection on statistics for biologists contains articles on many of the points above.

\section{Software and code}

Policy information about availability of computer code

Data collection Luminex xPONENT Software, Biotek Gen5 software

Data analysis GraphPad Prism v8.4, Luminex xPonent v3.1, Biotek Gen5 v1.05, R v.3.5, FastQC v.0.11.2, cutadapt v.1.6, TopHat v.2.0.13, Bowtie v.1.1.1, Samtools v.1.1, HTSeq v.0.6, DESeq2 v.1.22.2, ClueGO v.2.5.5 GluePedia v.1.5.5 plugin of Cytoscape v.3.7.2, ggdendro v.0.1.21, ggcorrplot v.0.1.3 ggplot2 v.3.3.2

For manuscripts utilizing custom algorithms or software that are central to the research but not yet described in published literature, software must be made available to editors and

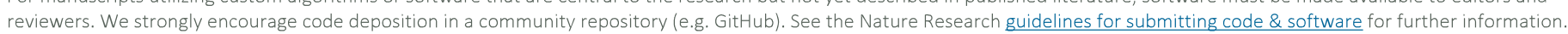

\section{Data}

Policy information about availability of data

All manuscripts must include a data availability statement. This statement should provide the following information, where applicable:

- Accession codes, unique identifiers, or web links for publicly available datasets

- A list of figures that have associated raw data

- A description of any restrictions on data availability

The raw RNAseq data concerning Fig. 3 and Fig. 4 have been deposited at GEO (http://www.ncbi.nlm.nih.gov/geo/) under BioProject accession number \# PRJNA638753 and are publicly available. 
Please select the one below that is the best fit for your research. If you are not sure, read the appropriate sections before making your selection.

\ Life sciences

Behavioural \& social sciences

Ecological, evolutionary \& environmental sciences

For a reference copy of the document with all sections, see nature.com/documents/nr-reporting-summary-flat.pdf

\section{Life sciences study design}

All studies must disclose on these points even when the disclosure is negative.

Sample size Sample size was determined according to the maximal availability of COVID-19 and Flu patients' sample sets where detailed clinical and laboratory data, as well as serological material were available.

Data exclusions There was no exclusion of data. All samples with available data were included in the respective analyses.

Replication All serum measurements were done with standardized commercially available kits. Patient serum samples was analyzed with different lots of kits always in the presence of internal controls to ensure consistency of the assays and accuracy of the measurements. In addition, several measurements ( $n=25$ samples) were replicated independently, at different times and with different lots of kits. No significant variability between measurements of the same samples was observed.

Randomization According to the clinical characteristics (severity of disease, timecourse of disease). Covariates such as age and gender were similar between hospitalized COVID-19 and flu patients, as well as their critically and non-critically ill subgroups.

Blinding Investigators were blinded to group allocation during data collection. Also, cytokine measurements were done by investigatora blinded to the patient information and clinical disease.

\section{Reporting for specific materials, systems and methods}

We require information from authors about some types of materials, experimental systems and methods used in many studies. Here, indicate whether each material, system or method listed is relevant to your study. If you are not sure if a list item applies to your research, read the appropriate section before selecting a response.

\begin{tabular}{|c|c|c|c|}
\hline \multicolumn{2}{|c|}{ Materials \& experimental systems } & \multicolumn{2}{|c|}{ Methods } \\
\hline $\mathrm{n} / \mathrm{a}$ & Involved in the study & $\mathrm{n} / \mathrm{a}$ & Involved in the study \\
\hline$\bigotimes$ & $\square$ Antibodies & Х & $\square$ ChIP-seq \\
\hline Х & $\square$ Eukaryotic cell lines & Х & $\square$ Flow cytometry \\
\hline$\bigotimes$ & $\square$ Palaeontology and archaeology & Х & $\square$ MRI-based neuroimaging \\
\hline Х & $\square$ Animals and other organisms & & \\
\hline - & $\bigotimes$ Human research participants & & \\
\hline Х & $\square$ Clinical data & & \\
\hline Х & $\square$ Dual use research of concern & & \\
\hline
\end{tabular}

\section{Human research participants}

Policy information about studies involving human research participants

Population characteristics

Patients with documented SARS-CoV2 infection, patients with documented flu infection, healthy individuals. Their detailed characteristics are provided in the manuscript as Supplementary Tables 1 and 2. For healthy individuals, 3/10 patients (30\%) were male. Median age was 54.5 (41-70) years.

Recruitment

All patients (i) admitted and hospitalized for community acquired pneumonia due to COVID-19 or FLU at the specific clinic/ ICU of Sotiria and Attikon Hospitals, (ii) admitted with FLU but non-hospitalized (as detailed in the manuscript) were recruited. There was no selection bias.

Ethics oversight

The study conforms to the principles outlined in the Declaration of Helsinki, and received approval by the Ethics Committees of the "Sotiria" General Chest Diseases Hospital, Athens, Greece (Approval numbers 16707/10-7-18 and 8385/31-3-20) and the "Attikon" University Hospital, University of Athens Medical School, Athens, Greece (Approval number 1821A/22-9-16). Written informed consent was provided by all participants. 